\title{
Correlation of Polynuclear Aromatic Hydrocarbon Formation Between Pyrolysis and Smoking*
}

\author{
by R. F. Severson, W. S. Schlotzbauer, R. F. Arrendale, M. E. Snook, and H. C. Higman \\ Tobacco and Health Laboratory, Agricultural Research Service, United States Department of Agriculture, \\ Athens, Georgia, U.S.A.
}

\section{INTRODUCTION}

Currently, there is considerable interest in the relationship between tobacco constituents and the polynuclear aromatic hydrocarbons (PAH) of tobacco smoke. Since it is well documented that at high temperatures organic materials produce $\operatorname{PAH}(1,2,3)$, numerous pyrolytic studies have shown that the hexane or petroleum ether (PE) extract of tobacco contains major precursors of smoke PAH $(4,5,6)$. Since the hexane extract is composed of numerous classes of organic compounds $(7,8)$, much controversy has arisen over which compound and/or classes of compounds in the extract are the most efficient $\mathrm{PAH}$ producers (9, $10,11)$.

Many of the studies have been criticized because conditions differing from actual smoking were employed (11). We felt that such criticism could be minimized if one would use pyrolytic conditions which would produce tobacco pyrolyzate PAH distributions similar to those found in cigarette smoke condensate (CSC). By applying recently developed methods for obtaining smoke PAH profile data $(12,13,14)$, it appeared possible to determine pyrolytic conditions which yield correlatable pyrolyzate-CSC PAH profiles. The use of these conditions in a systematic search for smoke PAH precursors should allow determination of the contribution of the various leaf constituents to the total PAH content of tobacco smoke. We, therefore, studied the effects of different pyrolysis temperatures on PAH levels produced by whole tobacco, its PE extract, and the residual extracted tobacco (marc). We compared the gas chromatographic PAH profiles of the pyrolyzates with those of CSC to determine the optimum pyrolytic temperature. Results of these experiments are reported, as are yields of phenols and acids in the pyrolyzates.

\footnotetext{
* Received for publication: 16th March, 1976.
}

\section{EXPERIMENTAL}

\section{Petroleum Ether Extraction}

A sample $(335 \mathrm{~g})$ of finely ground (16 mesh) 1971 South Carolina flue-cured tobacco (redried, unaged) was extracted with petroleum ether (PE) (boiling point $30-60^{\circ} \mathrm{C}$ ) in a Soxhlet apparatus for $64 \mathrm{~h}$. The extract was subsequently filtered and taken to dryness on a rotary evaporator to yield $26.5 \mathrm{~g}(7.9 \%$ yield $)$ of material. The extracted tobacco (marc) was removed from the extraction thimble and oven-dried $\left(90^{\circ} \mathrm{C}\right.$, $5 \mathrm{~h}$ ) to a weight of $308.5 \mathrm{~g}$. Portions of the marc and PE extract were used for pyrolytic studies.

\section{Solvents}

All solvents used were glass-distilled Burdick and Jackson $^{\text {** }}$ type and were redistilled prior to use.

\section{Pyrolysis}

The pyrolyses were performed in a horizontal $5 \mathrm{ft} . \times 2$ in. inside diameter Vycor tube inserted into a Lindberg Heavy-Duty furnace. Nitrogen flow through the pyrolysis system was maintained at $150 \mathrm{ml} / \mathrm{min}$. The oven was pre-heated to the desired temperature $(650$, $700,750,800,850 \pm 5^{\circ} \mathrm{C}$ at the center of the heated zone). The sample $(5 \mathrm{~g}$ of PE extract, $10 \mathrm{~g}$ of tobacco or marc) contained in a Vycor boat was placed at the cool end of the pyrolysis tube. The boat was drawn in the direction of the gas flow at a rate of $33.97 \mathrm{~cm} / \mathrm{min}$ by a wire attached to a kymograph (gear driven rotating drum) into the center of the heated zone. Products were flushed from the tube by the nitrogen into traps consisting of an uncooled 51 expansion flask, an ice-water-cooled cold finger, two dry ice-cooled cold

7* Reference to a company or product name does not imply approval or recommendation by the USDA. 
fingers, and a gas-washing bottle containing ethyl ether (E)/benzene (B) $(1: 1, \nabla / v)$ and $0.5 \mathrm{~N} \mathrm{N2OH}$, in a $1: 1$ volume ratio.

\section{Fractionation of Pyrolyzates}

After quantitative removal of the products from the traps with the E-B solvent, the pyrolyzates were fractionated as outlined in Figure 1. The pyrolyzates were partitioned between the E-B solvent and $1 \mathrm{~N} \mathrm{NaOH}$. The aqueous layer was adjusted to $\mathrm{pH} 6.5$ with concentrated $\mathrm{HCl}$ and phenolics were extracted with $\mathrm{E}$. The aqueous layer was further adjusted to $\mathrm{pH} 2.0$ and the acids were extracted with $E$.

The neutral fraction (NF) was divided into two portions representing $10 \%$ and $90 \%$ of the solution. The one-tenth portion was taken to constant weight for determination of the NF yield. The larger portion was reduced in volume and slurried with $40 \mathrm{~g}$ of silicic acid (SA) [pre-washed with methanol (M), and activated at $150{ }^{\circ} \mathrm{C}$ overnight] and $100 \mathrm{ml}$ each of B and isooctane. Benzene and excess isooctane were removed on a rotary evaporator until only an isooctanewet, SA-sample mixture remained. The NF-SA mixture was slurried with $P E$ and poured onto a $100 \mathrm{~g} \mathrm{SA}$ column. The column was eluted with a total of $500 \mathrm{ml}$ of $\mathrm{PE}$ (which included the amount used for slurrying the NF-SA sample) to yield fraction F-PE. Paraffins, which could interfere with PAH analyses were eluted by the PE (15). The PAH-containing fraction (F-BPE) was eluted with $1500 \mathrm{ml}$ of $25 \% \mathrm{~B}$ in $75 \% \mathrm{PE}$.
Figure 2. UV absorption curves for eliutions of F-BPE and standards used In determination of limits for CF cuts GF-B and GF-C.

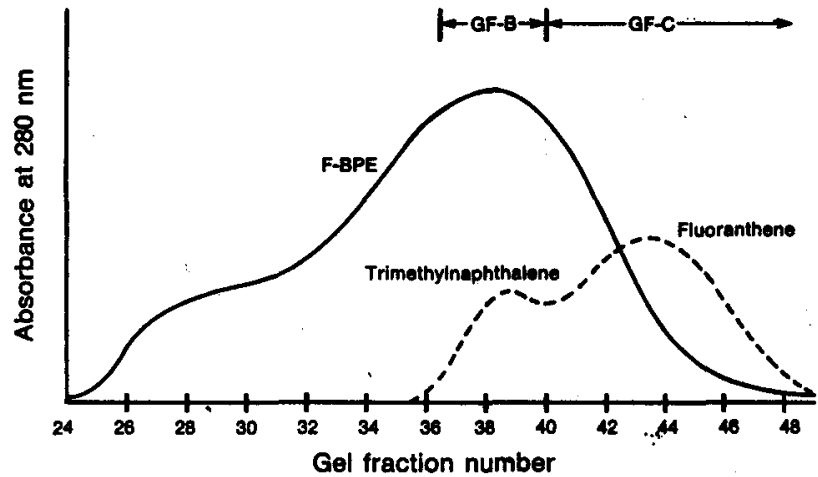

Elutions with $1000 \mathrm{ml}$ volumes of $B, E$, and $M$ yielded F-B, F-E, and F-M, respectively. The yields of NF and its subfractions are listed in Table 1.

The PAH were isolated from F-BPE by the Bio-Beads SX-12 gel filtration (GF) system, previously detailed $(12,13)$. For the pyrolyzates of the PE extract, concentrated F-BPE solutions in B were diluted to $10 \mathrm{ml}$. A $1 \mathrm{ml}$ sample of each of these solutions was placed onto the GF system, and $2 \mathrm{ml}$ were evaporated to dryness for determination of fraction weight. The F-BPE solutions of tobacco and marc pyrolyzates were transferred to $2 \mathrm{ml}$ volumetric flasks. A $1 \mathrm{ml}$ portion was placed on the GF system and the remainder was used for determination of fraction yield.

PAH in GF fractions were collected in two portions as determined by the elution volumes of trimethyl-

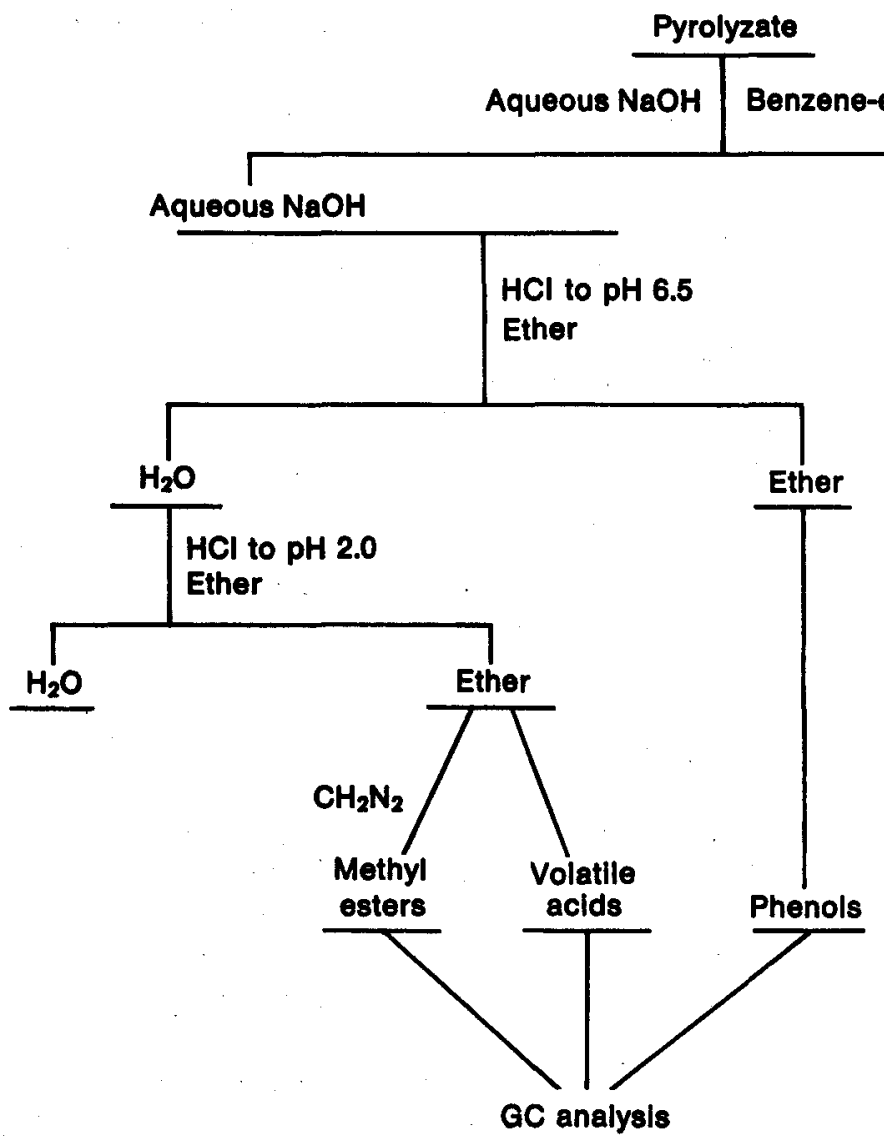

Figure 1. Pyrolyzate fractionation scheme. 
Table 1. Effect of pyrolysls temperature on the ylelds of chromatographlc fractions from pyrolyzate neutrals.

\begin{tabular}{|c|c|c|c|c|c|c|c|c|c|}
\hline \multirow{2}{*}{ Fraction } & \multicolumn{5}{|c|}{ PE extract } & \multicolumn{3}{|c|}{ Tobacco } & \multirow{2}{*}{ Marc } \\
\hline & $650^{\circ} \mathrm{C}$ & $700^{\circ} \mathrm{C}$ & $750^{\circ} \mathrm{C}$ & $800^{\circ} \mathrm{C}$ & $850^{\circ} \mathrm{C}$ & $650^{\circ} \mathrm{C}$ & $700^{\circ} \mathrm{C}$ & $750^{\circ} \mathrm{C}$ & \\
\hline & & & & Yieid (g) & $00 \mathrm{~g}$ pyro & rzed)a & & & \\
\hline \multirow[t]{2}{*}{ Neutrals } & 24.84 & 22.27 & 26.19 & 28.17 & 24.43 & 6.19 & 5.15 & 5.08 & 4.71 \\
\hline & \multicolumn{9}{|c|}{$\%$ by weight of neutralsb } \\
\hline Petroleum ether (PE) & 10.2 & 10.6 & 13.1 & 6.9 & 7.8 & 7.3 & 7.3 & 7.5 & 3.1 \\
\hline Benzene-petroleum ether (BPE) & 51.3 & 62.5 & 50.6 & 59.5 & 72.8 & 16.0 & 25.9 & 31.3 & 6.6 \\
\hline Benzene (B) & 5.6 & 6.8 & 8.7 & 8.8 & 14.1 & 14.2 & 9.9 & 14.1 & 10.8 \\
\hline Ethyl ether (E) & 15.8 & 16.2 & 14.5 & 7.7 & 9.5 & 28.1 & 22.9 & 25.4 & 4.5 \\
\hline Methanol (M) & 7.2 & 6.8 & 6.0 & 4.1 & 4.4 & 22.8 & 21.6 & 19.7 & 28.3 \\
\hline Total & 90.1 & 102.3 & 92.9 & 87.0 & 108.6 & 88.4 & 87.6 & 98.0 & 53.3 \\
\hline
\end{tabular}

a: Based on one determination.

b: Based on total weight of neutral placed on sllicic acid column.

naphthalene (GF fractions 36-39, portion GF-B) and fluoranthene (GF fractions 40-60, portion GF-C) (Figure 2).

\section{Cigarette Smoke Condensate PAH Isolation}

The Kentucky 1R1 Reference cigarettes were smoked under standard conditions $(16,17)$. The PAH in the CSC from 90 cigarettes were isolated according to our procedure (13). Thus, the CSC dissolved in B-M-E was extracted with water, and organic solubles subjected to SA column and GF chromatography as described above to yield PAH isolates GF-B and GF-C.

\section{Gas Chromatographic (GC) Analysis}

The PAH in GF-B and GF-C were analyzed on a Hewlett-Padkard 5830 reporting gas chromatograph equipped with a $15 \mathrm{ft}$. $\times 1 / 8$ in. stainless steel column padked with $5 \%$ Dexsil 300 GC on 100/120 mesh Chromosorb W-AW. Helium flow rate was $40 \mathrm{ml} / \mathrm{min}$ (measured at $90^{\circ} \mathrm{C}$ ). Oven temperature was held for $10 \mathrm{~min}$ at $90^{\circ} \mathrm{C}$ after sample injection, increased to $325{ }^{\circ} \mathrm{C}$ at $2 \% / \mathrm{min}$, then held at $325{ }^{\circ} \mathrm{C}$ for $45 \mathrm{~min}$.
The injection port and flame ionization detector were maintained at 290 and $350^{\circ} \mathrm{C}$, respectively. Response data were obtained for the major parent PAH. Hexatriacontane $\left(\mathrm{C}_{36}\right)$ and pentatriacontane $\left(\mathrm{C}_{95}\right)$ were used as internal standards for PAH quantitation of pyrolyzates and CSC, respectively.

The gas chromatograms of all GF-B and GF-C portions were divided into the following fractions by retention times: naphthalene, relative retention time (RRT) 0.238-0.568; fluorene, RRT 0.581-0.731; phenanthrene, RRT 0.754-0.951; pyrene, RRT 0.964-1.180; chrysene, RRT 1.203-1.359; and benzopyrene, RRT 1.384-1.594. The total peak areas of each fraction from the two chromatograms were combined. Assuming detector responses identical to those of the major parent $\mathrm{PAH}$, GC volatile PAH weights were then calculated for each fraction (Table 2).

Phenols were analyzed on a $6 \mathrm{ft} . \times 1 / 8$ in. glass column containing $10 \%$ SP-2401 on 100/120 mesh Supelcoport. The injector and flame ionization detector were maintained at $215{ }^{\circ} \mathrm{C}$. After a pre-hold at $40^{\circ} \mathrm{C}$ for $3 \mathrm{~min}$, the oven was temperature programmed to $200^{\circ} \mathrm{C}$ at $4^{\circ} / \mathrm{min}$. The helium flow rate was $35 \mathrm{ml} / \mathrm{min}$.

Table 2. Total GC PAH traction welghts of pyrolyzate PAH.

\begin{tabular}{|c|c|c|c|c|c|c|c|c|c|}
\hline \multirow{2}{*}{ PAH group } & \multicolumn{5}{|c|}{ PE extract } & \multicolumn{3}{|c|}{ Tobacco } & \multirow{2}{*}{ Marc } \\
\hline & $650^{\circ} \mathrm{C}$ & $700^{\circ} \mathrm{C}$ & $750^{\circ} \mathrm{C}$ & $800^{\circ} \mathrm{C}$ & $850^{\circ} \mathrm{C}$ & $650^{\circ} \mathrm{C}$ & $700^{\circ} \mathrm{C}$ & $750^{\circ} \mathrm{C}$ & \\
\hline & \multicolumn{9}{|c|}{ Amount $(g / 100 g)^{a}$} \\
\hline Naphthalene & 3.08 & 4.88 & 4.29 & 4.45 & 4.70 & 0.20 & 0.32 & 1.31 & 0.14 \\
\hline Fluorene & 1.04 & 1.41 & 1.28 & 1.02 & 0.62 & 0.14 & 0.11 & 0.26 & 0.087 \\
\hline Phenanthrene & 1.25 & 1.89 & 2.32 & 3.05 & 2.60 & 0.17 & 0.16 & 0.32 & 0.090 \\
\hline Pyrene & 0.68 & 1.03 & 1.39 & 1.88 & 1.85 & 0.072 & 0.063 & 0.18 & 0.040 \\
\hline Chrysene & 0.20 & 0.33 & 0.55 & 0.94 & 0.90 & 0.017 & 0.018 & 0.070 & 0.009 \\
\hline Benzopyrene & 0.13 & 0.17 & 0.47 & 1.21 & 1.51 & 0.003 & 0.019 & 0.053 & 0.006 \\
\hline Total & 6.38 & 9.71 & 10.30 & 12.55 & 12.18 & 0.60 & 0.69 & 2.19 & 0.33 \\
\hline$\%$ Benzene-petroleum ether (BPE) & 25.7 & 43.6 & 39.5 & 44.8 & 55.4 & 10.6 & 48.0 & 48.0 & 106.0 \\
\hline
\end{tabular}

a: Based on GC volatlle material assuming detector response for group is identlcal to that of major parent PAH; average of two determinations $( \pm 5 \%$. 
Table 3. Comparison of PAH dlstributions in gel filtration cut GF-C of CSC and pyrolyzates.

\begin{tabular}{|c|c|c|c|c|c|c|c|}
\hline \multirow{3}{*}{$\begin{array}{l}\text { Peak } \\
\text { No. }\end{array}$} & \multirow{3}{*}{$\begin{array}{l}\text { Relative } \\
\text { retention } \\
\text { timea }\end{array}$} & \multirow{3}{*}{ Compound } & \multirow[b]{2}{*}{ csc } & \multicolumn{4}{|c|}{ Pyrolyzate } \\
\hline & & & & $\begin{array}{l}700^{\circ} \mathrm{C} \\
\text { Tobacco }\end{array}$ & $\begin{array}{l}.700^{\circ} \mathrm{C} \\
\text { Marc }\end{array}$ & $\begin{array}{l}700^{\circ} \mathrm{C} \\
\mathrm{PE}\end{array}$ & $\begin{array}{l}\mathrm{B} 50^{\circ} \mathrm{C} \\
\mathrm{PE}\end{array}$ \\
\hline & & & \multicolumn{5}{|c|}{$\%$ Compositionb } \\
\hline 1 & 0.238 & naphthalene & 3.35 & 9.77 & 13.32 & 9.39 & 19.19 \\
\hline 2 & 0.292 & dimethylindenes & 1.22 & 0.32 & 0.48 & 1.14 & - \\
\hline 3 & 0.300 & $\begin{array}{l}\text { dimethylindenes } \\
\text { unidentified }\end{array}$ & $\underset{f}{1.03}$ & $\begin{array}{l}0.56 \\
c \\
d\end{array}$ & $\begin{array}{l}0.69 \\
c \\
d\end{array}$ & $\begin{array}{l}0.98 \\
c \\
d\end{array}$ & 一 \\
\hline 4 & 0.335 & 2-methylnaphthalene & 2.42 & 5.11 & 3.10 & 9.18 & 1.91 \\
\hline 5 & 0.350 & 1-methyinaphthalene & 2.98 & 5.09 & 3.48 & 6.75 & 1.27 \\
\hline 6 & 0.372 & $\begin{array}{l}\text { unidentified } \\
\text { trimethylindene }\end{array}$ & $\begin{array}{c}0.38 \\
f\end{array}$ & $\underset{f}{0.31}$ & 0.30 & $\stackrel{0.54}{f}$ & - \\
\hline 7 & 0.396 & $\begin{array}{l}\text { biphenyl } \\
\text { trimethylindene }\end{array}$ & $\begin{array}{c}0.47 \\
\mathbf{C} \\
\mathbf{e}\end{array}$ & $\begin{array}{l}0.26 \\
\mathrm{c} \\
\mathrm{e}\end{array}$ & $\underset{f}{0.23}$ & $\begin{array}{l}0.54 \\
\mathrm{c} \\
e\end{array}$ & $\frac{1.27}{f}$ \\
\hline 8 & 0.434 & $\begin{array}{l}\text { dimethylnaphthalenes } \\
1 \text {-vinylnaphthalene }\end{array}$ & $\begin{array}{l}1.09 \\
c \\
d\end{array}$ & $\begin{array}{l}0.57 \\
\text { c } \\
\text { d }\end{array}$ & $\begin{array}{l}0.59 \\
\mathbf{C} \\
\mathbf{C}\end{array}$ & $\begin{array}{l}2.55 \\
c \\
d\end{array}$ & $\begin{array}{c}0.21 \\
f\end{array}$ \\
\hline 9 & 0.450 & $\begin{array}{l}\text { dimethylnaphthalenes } \\
\text { 2-vinylnaphthalene }\end{array}$ & $\begin{array}{l}2.95 \\
\mathrm{C} \\
\mathrm{C}\end{array}$ & $\begin{array}{l}2.55 \\
\mathrm{C} \\
\mathrm{C}\end{array}$ & $\begin{array}{l}2.05 \\
\text { c } \\
d\end{array}$ & $\begin{array}{c}5.68 \\
c \\
c\end{array}$ & $\begin{array}{c}1.28 \\
f\end{array}$ \\
\hline 10 & 0.467 & dimethylnaphthalenes & 1.12 & 0.55 & 0.56 & 1.36 & - \\
\hline 11 & 0.476 & $\begin{array}{l}\text { acenaphthylene } \\
\text { dimethylnaphthalene }\end{array}$ & $\begin{array}{l}2.16 \\
\mathrm{C} \\
\mathrm{C}\end{array}$ & $\begin{array}{c}8.71 \\
c \\
e\end{array}$ & $\underset{f}{6.64}$ & $\begin{array}{c}3.78 \\
c \\
\theta\end{array}$ & 6.54 \\
\hline 12 & 0.503 & $\begin{array}{l}\text { acenaphthene } \\
\text { dimethylnaphthalene }\end{array}$ & $\begin{array}{l}0.70 \\
\text { c } \\
\text { c }\end{array}$ & $\begin{array}{l}0.50 \\
\text { C } \\
\text { e }\end{array}$ & $\underset{f}{0.54}$ & $\begin{array}{l}0.56 \\
c \\
\theta\end{array}$ & $\underset{f}{0.41}$ \\
\hline 13 & 0.526 & $\begin{array}{l}\text { trimethyinaphthalene } \\
\text { dibenzfuran }\end{array}$ & $\begin{array}{l}1.38 \\
\text { d } \\
\text { c }\end{array}$ & $\begin{array}{l}0.64 \\
\text { d } \\
c\end{array}$ & $\begin{array}{c}0.87 \\
0 \\
c\end{array}$ & $\underset{f}{0.31}$ & $\begin{array}{c}0.09 \\
f\end{array}$ \\
\hline 14 & 0.545 & $\begin{array}{l}\text { trimethylnaphthalene } \\
\text { naphthofuran }\end{array}$ & $\begin{array}{c}0.46 \\
e \\
c\end{array}$ & $\begin{array}{c}0.79 \\
e \\
c\end{array}$ & $\begin{array}{c}0.85 \\
e \\
c\end{array}$ & $\begin{array}{c}0.49 \\
c \\
d\end{array}$ & $\begin{array}{c}0.06 \\
f\end{array}$ \\
\hline 15 & 0.555 & $\begin{array}{l}\text { methyl-2-vinylnaphthalene } \\
\text { trimethylnaphthalenes }\end{array}$ & 1.21 & $\begin{array}{l}0.68 \\
\mathbf{C} \\
\mathrm{e}\end{array}$ & $\begin{array}{c}0.79 \\
\mathbf{C} \\
\text { e }\end{array}$ & $\begin{array}{c}1.07 \\
c \\
d\end{array}$ & - \\
\hline 16 & 0.568 & $\begin{array}{l}\text { methyl-2-vinylnaphthalene } \\
\text { trimethylnaphthalene }\end{array}$ & $\begin{array}{c}0.80 \\
e \\
c\end{array}$ & $\begin{array}{c}0.20 \\
\mathrm{C} \\
\mathrm{C}\end{array}$ & $\begin{array}{c}0.17 \\
c \\
d\end{array}$ & $\begin{array}{c}0.80 \\
c \\
c\end{array}$ & - \\
\hline 17 & 0.581 & 1-methylacenaphthylene & $\stackrel{1.36}{c}$ & 2.32 & 2.10 & 1.64 & 0.21 \\
\hline 18 & 0.588 & $\begin{array}{l}\text { fluorene } \\
\text { methylacenaphthylene }\end{array}$ & $\begin{array}{c}5.19 \\
c \\
d\end{array}$ & $\begin{array}{c}4.09 \\
c \\
c\end{array}$ & $\begin{array}{c}3.69 \\
\mathrm{C} \\
\mathrm{C}\end{array}$ & $\begin{array}{c}2.80 \\
\mathrm{C} \\
\mathrm{C}\end{array}$ & $\begin{array}{c}2.45 \\
\text { C } \\
\theta\end{array}$ \\
\hline
\end{tabular}


Table 3 (continued)

\begin{tabular}{|c|c|c|c|c|c|c|c|c|}
\hline \multirow{3}{*}{$\begin{array}{l}\text { Peak } \\
\text { No. }\end{array}$} & \multirow{3}{*}{$\begin{array}{l}\text { Relative } \\
\text { retention } \\
\text { timea }\end{array}$} & \multirow{3}{*}{ Compound } & & \multirow[b]{2}{*}{ CSC } & \multicolumn{4}{|c|}{ Pyrolyzate } \\
\hline & & & & & $\begin{array}{l}700^{\circ} \mathrm{C} \\
\text { Tobacco }\end{array}$ & $\begin{array}{l}700^{\circ} \mathrm{C} \\
\text { Marc }\end{array}$ & $\underset{\mathrm{PE}}{700^{\circ} \mathrm{C}}$ & $\begin{array}{l}850^{\circ} \mathrm{C} \\
\mathrm{PE}\end{array}$ \\
\hline & & & & \multicolumn{5}{|c|}{$\%$ Compositionb } \\
\hline 19 & 0.596 & $\begin{array}{l}\text { methylacenaphthylene } \\
\text { 9-methylfluorene }\end{array}$ & & $\begin{array}{l}1.00 \\
c \\
c\end{array}$ & $\underset{f}{1.57}$ & $\underset{f}{1.44}$ & $\stackrel{1.17}{f}$ & $\underset{f}{0.01}$ \\
\hline 20 & 0.612 & $\begin{array}{l}\text { methylacenaphthene } \\
\text { benz }(\theta) \text { indene }\end{array}$ & & $\begin{array}{l}1.58 \\
c \\
c\end{array}$ & $\begin{array}{l}1.35 \\
\mathrm{c} \\
\mathrm{c}\end{array}$ & $\begin{array}{l}1.51 \\
c \\
c\end{array}$ & $\begin{array}{l}1.16 \\
c \\
d\end{array}$ & $\begin{array}{r}0.07 \\
f\end{array}$ \\
\hline 21 & 0.629 & methylacenaphthene & & $\begin{array}{c}0.22 \\
d\end{array}$ & $\stackrel{1.09}{f}$ & $\underset{f}{0.99}$ & $\underset{f}{0.57}$ & $\underset{f}{0.01}$ \\
\hline 22 & 0.636 & benz(f)indene & & 0.93 & 1.78 & 1.88 & 0.79 & 0.19 \\
\hline 23 & 0.647 & $\begin{array}{l}\text { methylnaphthofuran } \\
\text { dimethylacenaphthene }\end{array}$ & & $\begin{array}{l}0.17 \\
c \\
d\end{array}$ & $\begin{array}{l}0.05 \\
c \\
d\end{array}$ & $\underset{f}{0.56}$ & $\begin{array}{l}0.09 \\
d \\
c\end{array}$ & - \\
\hline 24 & 0.660 & $\begin{array}{l}\text { methylnaphthofuran } \\
\text { dimethylacenaphthene }\end{array}$ & & $\begin{array}{l}0.24 \\
c \\
d\end{array}$ & $\begin{array}{l}0.07 \\
c \\
d\end{array}$ & $\begin{array}{l}0.34 \\
\text { c } \\
d\end{array}$ & $\begin{array}{l}0.36 \\
d \\
c\end{array}$ & 一 \\
\hline 25 & 0.682 & $\begin{array}{l}\text { 2- \& 3-methylfluorene } \\
\text { methylnaphthofuran } \\
\text { dimethylacenaphthene }\end{array}$ & & $\begin{array}{l}3.56 \\
c \\
d \\
d\end{array}$ & $\begin{array}{l}1.37 \\
c \\
d \\
d\end{array}$ & $\begin{array}{l}1.62 \\
c \\
d \\
d\end{array}$ & $\begin{array}{l}1.84 \\
c \\
f \\
d\end{array}$ & $\begin{array}{c}0.28 \\
c \\
c\end{array}$ \\
\hline 26 & 0.694 & $\begin{array}{l}\text { 1- \& 4-methylfluorene } \\
\text { dimethylacenaphthene } \\
\text { methyldibenzofuran }\end{array}$ & & $\begin{array}{l}2.99 \\
c \\
d \\
e\end{array}$ & $\begin{array}{l}0.96 \\
c \\
d \\
e\end{array}$ & $\begin{array}{l}1.19 \\
c \\
d \\
c\end{array}$ & $\begin{array}{l}1.44 \\
c \\
c \\
e\end{array}$ & $\begin{array}{c}0.31 \\
\mathrm{C} \\
\mathrm{c}\end{array}$ \\
\hline 27 & 0.704 & $\begin{array}{l}\text { methylbenz(e)indene } \\
\text { dimethylacenaphthylene } \\
\text { dimethylacenaphthene }\end{array}$ & & $\begin{array}{l}0.85 \\
\text { c } \\
\text { d } \\
\text { d }\end{array}$ & $\begin{array}{l}0.63 \\
c \\
c \\
d\end{array}$ & $\begin{array}{l}0.85 \\
c \\
c \\
d\end{array}$ & $\begin{array}{l}0.97 \\
c \\
c \\
d\end{array}$ & - \\
\hline 28 & 0.716 & $\begin{array}{l}\text { methylbenz }(f) \text { indene } \\
\text { methylbenz }(e) \text { indene } \\
\text { dimethylacenaphthene }\end{array}$ & & $\begin{array}{l}1.13 \\
c \\
e \\
d\end{array}$ & $\begin{array}{l}0.97 \\
\text { c } \\
\text { c } \\
\text { d }\end{array}$ & $\begin{array}{l}1.24 \\
c \\
c \\
d\end{array}$ & $\begin{array}{c}1.22 \\
c \\
c \\
c\end{array}$ & $\begin{array}{l}0.07 \\
\text { c } \\
\text { d }\end{array}$ \\
\hline 29 & 0.731 & $\begin{array}{l}\text { methylbenz(f)indene } \\
\text { dimethylacenaphthene }\end{array}$ & & $\begin{array}{l}1.29 \\
c \\
d\end{array}$ & $\begin{array}{l}0.45 \\
c \\
d\end{array}$ & $\begin{array}{l}0.71 \\
c \\
d\end{array}$ & $\begin{array}{l}0.56 \\
c \\
d\end{array}$ & - \\
\hline 30. & 0.754 & phenanthrene & & 5.73 & 7.91 & 7.90 & 4.16 & 17.38 \\
\hline 31 & 0.760 & anthracene & . & 2.06 & 1.73 & - & 1.15 & - \\
\hline 32 & 0.843 & 2- \& 3-methylphenanthrene & & 5.23 & 3.33 & 2.65 & 3.44 & 1.39 \\
\hline 33 & 0.859 & $\begin{array}{l}\text { 1- \& 9-methylphenanthrene } \\
\text { 1- \& 2-methylanthracene }\end{array}$ & & $\begin{array}{l}3.88 \\
c \\
d\end{array}$ & $\begin{array}{l}4.87 \\
c \\
d\end{array}$ & $\begin{array}{l}4.21 \\
\mathrm{c} \\
\mathrm{C}\end{array}$ & $\begin{array}{l}3.91 \\
c \\
d\end{array}$ & $\begin{array}{l}1.98 \\
c \\
d\end{array}$ \\
\hline 34 & 0.877 & $\begin{array}{l}\text { 9-methylanthracene } \\
\text { 4-methylphenanthrene } \\
\text { 2-phenylnaphthalene }\end{array}$ & & $\begin{array}{c}0.43 \\
\text { C } \\
c\end{array}$ & $\begin{array}{l}0.71 \\
c \\
d \\
d\end{array}$ & $\begin{array}{l}0.91 \\
c \\
d \\
d\end{array}$ & $\begin{array}{l}0.50 \\
c \\
d \\
d\end{array}$ & $\begin{array}{c}0.88 \\
f\end{array}$ \\
\hline 35 & 0.905 & $\begin{array}{l}\text { dimethylphenanthrene } \\
\text { dimethylanthracene } \\
\text { unidentified }\end{array}$ & & $\begin{array}{l}0.61 \\
c \\
d\end{array}$ & $\begin{array}{c}0.23 \\
\mathrm{C} \\
\mathrm{c}\end{array}$ & $\begin{array}{c}0.55 \\
\mathrm{C} \\
\mathrm{c}\end{array}$ & $\begin{array}{c}0.05 \\
c \\
e\end{array}$ & $\begin{array}{c}0.03 \\
c \\
e\end{array}$ \\
\hline
\end{tabular}


Table 3. Comparison of PAH distributions in gel filtration cut GF-C of CSC and pyrolyzates (cont'd.).

\begin{tabular}{|c|c|c|c|c|c|c|c|}
\hline \multirow{3}{*}{$\begin{array}{l}\text { Peak } \\
\text { No. }\end{array}$} & \multirow{3}{*}{$\begin{array}{l}\text { Relative } \\
\text { retention } \\
\text { timea }\end{array}$} & \multirow{3}{*}{ Compound } & \multirow[b]{2}{*}{$\csc$} & \multicolumn{4}{|c|}{ Pyrolyzate } \\
\hline & & & & $\begin{array}{l}700^{\circ} \mathrm{C} \\
\text { Tobacco }\end{array}$ & $\begin{array}{l}700^{\circ} \mathrm{C} \\
\text { Marc }\end{array}$ & $\begin{array}{l}700^{\circ} \mathrm{C} \\
\mathrm{PE}\end{array}$ & $\begin{array}{l}850^{\circ} \mathrm{C} \\
\mathrm{PE}\end{array}$ \\
\hline & & & \multicolumn{5}{|c|}{$\%$ Compositionb } \\
\hline 36 & 0.924 & $\begin{array}{l}\text { dimethylphenanthrene } \\
\text { dimethylanthracene } \\
\text { unidentifledg }\end{array}$ & $\begin{array}{l}0.70 \\
c \\
d\end{array}$ & $\begin{array}{l}0.16 \\
c \\
d\end{array}$ & $\begin{array}{c}0.32 \\
c \\
d\end{array}$ & $\begin{array}{l}0.63 \\
c \\
d\end{array}$ & $\begin{array}{l}0.14 \\
c \\
d \\
c\end{array}$ \\
\hline 37 & 0.941 & $\begin{array}{l}\text { dimethylphenanthrene } \\
\text { dimethylanthracene } \\
\text { unidentifledg }\end{array}$ & 3.44 & $\begin{array}{l}2.04 \\
c \\
d\end{array}$ & $\begin{array}{l}1.99 \\
c \\
d\end{array}$ & $\begin{array}{c}2.61 \\
c \\
f\end{array}$ & $\begin{array}{c}0.26 \\
c \\
f \\
c\end{array}$ \\
\hline 38 & 0.951 & $\begin{array}{l}\text { dimethylphenanthrene } \\
\text { unidentifiedg }\end{array}$ & 0.84 & $\begin{array}{l}0.60 \\
c \\
d\end{array}$ & $\begin{array}{l}0.66 \\
c \\
d\end{array}$ & $\underset{f}{0.70}$ & $\begin{array}{c}0.11 \\
c \\
c\end{array}$ \\
\hline 39 & 0.964 & $\begin{array}{l}\text { fluoranthene } \\
\text { dimethylphenanthrene }\end{array}$ & $\begin{array}{l}2.58 \\
\mathrm{c} \\
\mathrm{e}\end{array}$ & $\begin{array}{l}1.70 \\
c \\
e\end{array}$ & $\begin{array}{l}1.58 \\
\mathrm{c} \\
\mathrm{e}\end{array}$ & $\begin{array}{c}1.14 \\
\mathrm{C} \\
\mathrm{e}\end{array}$ & $\begin{array}{l}4.95 \\
\mathrm{c} \\
\mathrm{e}\end{array}$ \\
\hline 40 & 0.973 & $\begin{array}{l}\text { acephenanthrylene } \\
\text { (aceanthrylene) }\end{array}$ & 0.95 & $\begin{array}{c}1.60 \\
c\end{array}$ & $\begin{array}{c}1.45 \\
c\end{array}$ & $\begin{array}{c}0.61 \\
c\end{array}$ & $\begin{array}{c}1.38 \\
c\end{array}$ \\
\hline 41 & 0.993 & di- or trimethylphenanthrene & 0.71 & 0.82 & 0.90 & 0.19 & 0.11 \\
\hline 42 & 1.000 & $\begin{array}{l}\text { pyrene } \\
\text { cyclopentenophenanthrene }\end{array}$ & $\begin{array}{c}2.90 \\
c \\
e\end{array}$ & $\begin{array}{l}3.17 \\
\mathrm{c} \\
\mathrm{e}\end{array}$ & $\underset{f}{2.83}$ & $\stackrel{2.22}{C}$ & $\begin{array}{c}7.40 \\
f\end{array}$ \\
\hline 43 & 1.032 & $\begin{array}{l}\text { trimethylphenanthrene(s) } \\
\text { cyclopentenophenanthrene }\end{array}$ & $\begin{array}{l}0.98 \\
c \\
c\end{array}$ & $\begin{array}{l}0.47 \\
\text { C } \\
\text { c. }\end{array}$ & $\begin{array}{c}0.79 \\
\mathbf{c} \\
\mathbf{c}\end{array}$ & $\begin{array}{l}0.41 \\
\mathrm{C} \\
\mathrm{c}\end{array}$ & 一 \\
\hline 44 & 1.044 & $\begin{array}{l}\text { 8-methylfluoranthene } \\
\text { trimethylphenanthrene }\end{array}$ & $\begin{array}{c}1.68 \\
c \\
e\end{array}$ & $\begin{array}{l}0.59 \\
c \\
e\end{array}$ & $\begin{array}{c}1.37 \\
c \\
\theta\end{array}$ & $\begin{array}{l}0.33 \\
c \\
\theta\end{array}$ & $\begin{array}{c}0.32 \\
c \\
e\end{array}$ \\
\hline 45 & 1.067 & $\begin{array}{l}\text { benzofluorene }(1,2 ; 2,3 ; 3,4) \\
\text { 1- \& 2-methylfluoranthene }\end{array}$ & $\begin{array}{l}2.67 \\
\mathrm{C} \\
\mathrm{c}\end{array}$ & $\begin{array}{l}2.54 \\
c \\
d\end{array}$ & $\begin{array}{l}2.41 \\
\mathbf{c} \\
\mathbf{d}\end{array}$ & $\begin{array}{l}1.01 \\
\text { c } \\
\text { d }\end{array}$ & $\begin{array}{l}1.59 \\
c \\
d\end{array}$ \\
\hline 46 & 1.080 & $\begin{array}{l}\text { 2-methylpyrene } \\
\text { trimethylphenanthrene }\end{array}$ & $\begin{array}{l}1.73 \\
c \\
c\end{array}$ & $\begin{array}{l}1.58 \\
\mathrm{C} \\
\mathrm{C}\end{array}$ & $\begin{array}{c}1.32 \\
c \\
c\end{array}$ & $\begin{array}{c}0.91 \\
\mathrm{C} \\
\mathrm{C}\end{array}$ & $\underset{f}{0.56}$ \\
\hline 47 & 1.095 & 1- \& 4-methylpyrene & $\begin{array}{c}2.26 \\
c\end{array}$ & $\underset{f}{2.44}$ & $\underset{f}{2.87}$ & $\underset{f}{1.70}$ & $\stackrel{1.05}{f}$ \\
\hline 48 & 1.124 & $\begin{array}{l}\text { methyl-1,2-benzofluorene } \\
\text { dimethylfluoranthene }\end{array}$ & $\begin{array}{c}0.61 \\
c \\
c\end{array}$ & $\begin{array}{l}0.20 \\
c \\
d\end{array}$ & $\begin{array}{l}0.50 \\
c \\
d\end{array}$ & $\begin{array}{l}0.36 \\
c \\
d\end{array}$ & $\underset{f}{0.12}$ \\
\hline 49 & 1.133 & $\begin{array}{l}\text { methylbenzofluorene } \\
\text { dimethylpyrene } \\
\text { dimethylfluoranthene }\end{array}$ & $\begin{array}{l}1.17 \\
\text { d } \\
\text { c } \\
\text { c }\end{array}$ & $\begin{array}{l}0.36 \\
c \\
d \\
d\end{array}$ & $\begin{array}{l}0.79 \\
c \\
d \\
e\end{array}$ & $\begin{array}{l}0.70 \\
\text { c } \\
d \\
d\end{array}$ & $\begin{array}{c}0.12 \\
\mathrm{c} \\
\mathrm{c}\end{array}$ \\
\hline 50 & 1.150 & $\begin{array}{l}\text { dimethylpyrene } \\
\text { dimethylfluoranthene } \\
\text { methylbenzofluorene }\end{array}$ & $\begin{array}{l}0.79 \\
c \\
d \\
d\end{array}$ & $\begin{array}{l}0.06 \\
c \\
d \\
c\end{array}$ & $\begin{array}{l}0.27 \\
c \\
d \\
c\end{array}$ & $\begin{array}{l}0.21 \\
c \\
c \\
d\end{array}$ & - \\
\hline 51 & 1.160 & $\begin{array}{l}\text { dimethylpyrene } \\
\text { benzo(c)phenanthrene } \\
\text { methylbenzofluorene }\end{array}$ & $\begin{array}{l}0.93 \\
c \\
e \\
d\end{array}$ & $\begin{array}{l}0.53 \\
c \\
e \\
c\end{array}$ & $\begin{array}{c}1.05 \\
c \\
e \\
c\end{array}$ & $\begin{array}{l}0.61 \\
c \\
d \\
e\end{array}$ & $\begin{array}{c}0.66 \\
f\end{array}$ \\
\hline 52 & 1.169 & $\begin{array}{l}\text { dimethylpyrenes } \\
\text { methylbenzofluorenes }\end{array}$ & 0.72 & $\begin{array}{l}0.16 \\
\text { c } \\
d\end{array}$ & $\begin{array}{c}0.49 \\
c \\
d\end{array}$ & $\begin{array}{l}0.34 \\
c \\
d\end{array}$ & - \\
\hline
\end{tabular}


Table 3 (continued)

\begin{tabular}{|c|c|c|c|c|c|c|c|}
\hline \multirow{3}{*}{$\begin{array}{l}\text { Peak } \\
\text { No. }\end{array}$} & \multirow{3}{*}{$\begin{array}{c}\text { Relative } \\
\text { retention } \\
\text { timea }\end{array}$} & \multirow{3}{*}{ Compound } & \multirow[b]{2}{*}{ csc } & \multicolumn{4}{|c|}{ Pyrolyzate } \\
\hline & & & & $\begin{array}{l}700^{\circ} \mathrm{C} \\
\text { Tobacco }\end{array}$ & $\begin{array}{l}700^{\circ} \mathrm{C} \\
\text { Marc }\end{array}$ & $\begin{array}{l}700^{\circ} \mathrm{C} \\
\mathrm{PE}\end{array}$ & ${ }_{\mathrm{PE}}^{850^{\circ} \mathrm{C}}$ \\
\hline & & & \multicolumn{5}{|c|}{$\%$ Compositionb } \\
\hline 53 & 1.180 & $\begin{array}{l}\text { dimethylpyrenes } \\
\text { benzo(g,h,i)fluoranthene }\end{array}$ & $\begin{array}{c}0.89 \\
c \\
d\end{array}$ & $\begin{array}{c}0.07 \\
c \\
\theta\end{array}$ & $\begin{array}{c}0.81 \\
c \\
e\end{array}$ & $\begin{array}{c}0.39 \\
c \\
e\end{array}$ & $\begin{array}{c}0.43 \\
f\end{array}$ \\
\hline 54 & 1.203 & 1,2-benzanthracene & 1.30 & 2.54 & 2.19 & 1.69 & 2.29 \\
\hline 55 & 1.207 & $\begin{array}{l}\text { chrysene } \\
\text { triphenylene }\end{array}$ & $\begin{array}{l}c \\
d\end{array}$ & $f$ & $f$ & f & $\begin{array}{l}3.49 \\
c \\
\theta\end{array}$ \\
\hline 56 & 1.230 & $\begin{array}{l}\text { 3,4-dimethylenepyrene } \\
\text { trimethylpyrene }\end{array}$ & $\begin{array}{l}0.11 \\
c \\
c\end{array}$ & $\begin{array}{c}0.08 \\
\mathrm{C} \\
\mathrm{C}\end{array}$ & $\begin{array}{l}0.35 \\
\mathbf{c} \\
\mathrm{c}\end{array}$ & $\begin{array}{l}0.17 \\
c \\
c\end{array}$ & 0.35 \\
\hline 57 & 1.276 & $\begin{array}{l}\text { methyl-1,2-benzanthracene } \\
\text { methylchrysene }\end{array}$ & $\begin{array}{l}0.53 \\
\mathrm{c} \\
\mathrm{c}\end{array}$ & $\begin{array}{l}0.86 \\
\mathrm{C} \\
\mathrm{C}\end{array}$ & $\begin{array}{c}0.72 \\
c \\
c\end{array}$ & $\begin{array}{l}1.10 \\
c \\
c\end{array}$ & $\begin{array}{l}0.60 \\
\mathbf{C} \\
\mathbf{c}\end{array}$ \\
\hline 58 & 1.288 & $\begin{array}{l}\text { methyl-1,2-benzanthracene } \\
\text { methylchrysene }\end{array}$ & $\begin{array}{l}0.08 \\
\mathrm{~d} \\
\mathrm{c}\end{array}$ & $\begin{array}{l}0.36 \\
\mathbf{c} \\
\mathbf{c}\end{array}$ & $\begin{array}{l}0.60 \\
\mathrm{c} \\
\mathrm{c}\end{array}$ & $\begin{array}{l}0.24 \\
\text { c } \\
c\end{array}$ & $\begin{array}{c}0.21 \\
\mathrm{C} \\
\mathrm{C}\end{array}$ \\
\hline 59 & 1.294 & methylchrysenes & $\underset{c}{0.11}$ & 0.50 & 0.94 & 0.39 & 0.73 \\
\hline 60 & 1.310 & $\begin{array}{l}\text { 3,4-trimethylenepyrene } \\
\text { dimethylchrysene }\end{array}$ & $\begin{array}{l}0.02 \\
c \\
d\end{array}$ & $\begin{array}{c}0.12 \\
c \\
e\end{array}$ & $\begin{array}{l}0.28 \\
c \\
d\end{array}$ & $\begin{array}{l}0.10 \\
c \\
e\end{array}$ & 一 \\
\hline 61 & 1.336 & $\begin{array}{l}\text { dimethylchrysene } \\
\text { dimethyl-1,2-benzanthracene }\end{array}$ & $\begin{array}{l}0.22 \\
c \\
c\end{array}$ & $\begin{array}{l}0.15 \\
\mathrm{c} \\
\mathrm{C}\end{array}$ & $\begin{array}{c}0.81 \\
\mathrm{c} \\
\mathrm{C}\end{array}$ & $\begin{array}{l}0.37 \\
c \\
c\end{array}$ & \\
\hline 62 & 1.359 & $\begin{array}{l}\text { dimethylchrysene } \\
\text { dimethyl-1,2-benzanthracene }\end{array}$ & $\begin{array}{l}0.13 \\
c \\
c\end{array}$ & $\begin{array}{l}0.13 \\
\mathrm{c} \\
\mathrm{C}\end{array}$ & $\begin{array}{c}0.09 \\
c \\
c\end{array}$ & $\begin{array}{l}0.35 \\
c \\
d\end{array}$ & \\
\hline 63 & 1.384 & benzo(b,j,k)fluoranthenes & 0.33 & 0.43 & 0.28 & 0.27 & 2.79 \\
\hline 64 & 1.401 & benzo(a)fluoranthene & 0.16 & 0.18 & 0.08 & 0.09 & 0.86 \\
\hline 65 & 1.429 & $\begin{array}{l}\text { benzo(e)pyrene } \\
\text { benzo(a)pyrene }\end{array}$ & $\begin{array}{l}0.34 \\
d \\
c\end{array}$ & $\begin{array}{l}0.75 \\
\mathrm{C} \\
\mathrm{C}\end{array}$ & $\begin{array}{l}0.54 \\
c \\
c\end{array}$ & $\begin{array}{c}0.57 \\
c \\
c\end{array}$ & $\begin{array}{c}3.78 \\
c \\
c\end{array}$ \\
\hline 66 & 1.449 & $\begin{array}{l}\text { perylene } \\
\text { methylbenzofluoranthene }\end{array}$ & $\begin{array}{c}0.04 \\
c \\
c\end{array}$ & $\begin{array}{l}0.16 \\
c \\
d\end{array}$ & $\begin{array}{c}0.29 \\
c \\
e\end{array}$ & $\begin{array}{c}0.31 \\
\mathrm{c} \\
\mathrm{e}\end{array}$ & $\stackrel{1.31}{f}$ \\
\hline 67 & 1.464 & methylbenzofluoranthenes & 0.02 & 0.07 & 0.12 & 0.24 & $1.00^{\circ}$ \\
\hline 68 & $\begin{array}{l}1.484- \\
1.492\end{array}$ & $\begin{array}{l}\text { methylbenzo(a)pyrene } \\
\text { methylbenzo(e)pyrene }\end{array}$ & $\begin{array}{c}0.10 \\
c \\
c\end{array}$ & $\begin{array}{l}0.29 \\
\mathrm{c} \\
\mathrm{c}\end{array}$ & $\begin{array}{c}0.10 \\
c \\
c\end{array}$ & $\begin{array}{c}0.32 \\
\mathbf{c} \\
\mathbf{c}\end{array}$ & $\begin{array}{l}1.06 \\
\mathrm{C} \\
\mathrm{c}\end{array}$ \\
\hline 69 & 1.508 & $\begin{array}{l}\text { methylbenzo(a)pyrene } \\
\text { methylbenzo(e)pyrene } \\
\text { methylperylene }\end{array}$ & $\begin{array}{c}0.09 \\
\text { c } \\
c \\
d\end{array}$ & $\begin{array}{l}0.32 \\
c \\
c \\
d\end{array}$ & $\begin{array}{l}0.06 \\
c \\
c \\
d\end{array}$ & $\begin{array}{l}0.36 \\
\text { c } \\
c \\
d\end{array}$ & $\begin{array}{l}0.47 \\
c \\
c \\
c\end{array}$ \\
\hline 70 & 1.541 & $\begin{array}{l}\text { methylbenzo(a)pyrene } \\
\text { methylbenzo(e)pyrene } \\
\text { methylperylene }\end{array}$ & $\begin{array}{l}0.06 \\
c \\
c \\
d\end{array}$ & $\begin{array}{l}0.04 \\
c \\
c \\
c\end{array}$ & $\begin{array}{c}0.02 \\
c \\
c \\
d\end{array}$ & $\begin{array}{l}0.08 \\
c \\
c \\
c\end{array}$ & $\begin{array}{l}0.15 \\
\mathrm{c} \\
\mathrm{C}\end{array}$ \\
\hline
\end{tabular}


Table 3. Comparison of PAH distributions in gel filtration cut GF-C of CSC and pyrolyzates (cont'd.).

\begin{tabular}{|c|c|c|c|c|c|c|c|}
\hline \multirow{3}{*}{$\begin{array}{l}\text { Peak } \\
\text { No. }\end{array}$} & \multirow{3}{*}{$\begin{array}{l}\text { Relative } \\
\text { retention } \\
\text { timen }\end{array}$} & \multirow{3}{*}{ Compound } & \multirow{2}{*}{$\csc$} & \multicolumn{4}{|c|}{ Pyrolyzate } \\
\hline & & & & $\begin{array}{l}700^{\circ} \mathrm{C} \\
\text { Tobacco }\end{array}$ & $\begin{array}{l}700^{\circ} \mathrm{C} \\
\text { Marc }\end{array}$ & $\begin{array}{c}700^{\circ} \mathrm{C} \\
\text { PE }\end{array}$ & $\begin{array}{c}850^{\circ} \mathrm{C} \\
\mathrm{PE}\end{array}$ \\
\hline & & & \multicolumn{5}{|c|}{$\%$ Compositionb } \\
\hline 71 & 1.551 & $\begin{array}{l}\text { methylbenzo(a)pyrene } \\
\text { methylbenzo(e)pyrene } \\
\text { indenofluoranthene } \\
\text { dibenzphenanthrene }\end{array}$ & - & $\begin{array}{c}0.02 \\
c \\
c \\
e \\
e\end{array}$ & $\begin{array}{c}0.03 \\
c \\
c \\
e\end{array}$ & $\begin{array}{l}0.03 \\
c \\
c \\
\theta \\
\theta\end{array}$ & $\begin{array}{l}0.06 \\
c \\
c \\
e \\
d\end{array}$ \\
\hline 72 & 1.586 & $\begin{array}{l}\text { dimethylbenzo(a)pyrene } \\
\text { dimethylbenzo(e)pyrene } \\
\text { dibenz(a,j)anthracene }\end{array}$ & - & $\begin{array}{l}0.04 \\
c \\
c \\
d\end{array}$ & 一 & $\begin{array}{l}0.05 \\
c \\
c \\
d\end{array}$ & 0.25 \\
\hline 73 & 1.594 & $\begin{array}{l}\text { dimethylbenzo(a)pyrene } \\
\text { dimethylbenzo(e)pyrene } \\
\text { dibenz(a,h)anthracene } \\
\text { dibenz(a,c)anthracene }\end{array}$ & - & $\begin{array}{l}0.10 \\
d \\
d \\
c \\
c\end{array}$ & $\begin{array}{c}0.02 \\
e \\
e \\
c \\
c\end{array}$ & $\begin{array}{l}0.04 \\
d \\
d \\
c \\
c\end{array}$ & $\begin{array}{l}0.58 \\
\text { c } \\
\text { c }\end{array}$ \\
\hline 74 & 1.612 & indenopyrene & 0.02 & 0.13 & 0.04 & 0.35 & 2.01 \\
\hline 75 & 1.691 & benzo(g,h,i)perylene & - & 0.12 & 0.08 & 0.11 & 0.30 \\
\hline 76 & 1.703 & $\begin{array}{l}\text { anthanthrene } \\
\text { methylindenopyrene }\end{array}$ & 一 & $\begin{array}{c}0.02 \\
\mathrm{c} \\
\mathrm{C}\end{array}$ & $\begin{array}{c}0.02 \\
c \\
d\end{array}$ & $\begin{array}{c}0.01 \\
c \\
c\end{array}$ & $\begin{array}{l}1.20 \\
c \\
c\end{array}$ \\
\hline 77 & 1.719 & $\begin{array}{l}\text { methylindenopyrene } \\
\text { methyl }(g, h, l) \text { perylene }\end{array}$ & - & $\begin{array}{l}0.01 \\
c \\
c\end{array}$ & - & $\begin{array}{c}0.01 \\
c \\
c\end{array}$ & $\begin{array}{l}0.56 \\
c \\
c\end{array}$ \\
\hline 78 & 1.751 & $\begin{array}{l}\text { methyl }(g, h, i) p e r y l e n e \\
\text { methylindenopyrene } \\
\text { dimethylindenopyrene }\end{array}$ & - & $\begin{array}{l}0.01 \\
c \\
c \\
d\end{array}$ & - & $\begin{array}{l}0.01 \\
c \\
c \\
d\end{array}$ & $\begin{array}{l}0.08 \\
c \\
c \\
d\end{array}$ \\
\hline 79 & 1.782 & $\begin{array}{l}\text { methyl }(g, h, i) \text { perylene } \\
\text { methylanthanthrene } \\
\text { dimethylindenopyrene }\end{array}$ & - & $\begin{array}{l}0.01 \\
c \\
d \\
c\end{array}$ & - & - & $\begin{array}{l}0.01 \\
c \\
d \\
c\end{array}$ \\
\hline 80 & 1.835 & dibenzpyrene & - & 0.07 & - & 0.04 & 0.04 \\
\hline
\end{tabular}

a: Relative to pyrene \pm 0.003 relative retention units.

b: Based on total peak area from RRT 0.238 through 1.835, assuming unity response.

6: Less than $5 \%$ of peak area (based on UV Intensity).

c: Greater than $30 \%$ of peak area (based on UV intensity).

$f:$ Only component appearing in UV spectra.

d: Accounts for 5 to $30 \%$ of peak area (based on UV intensity).

g: Possibly vinylphenanthrenes.

The volatile acids $\left(\mathrm{C}_{1}-\mathrm{C}_{7}\right)$ were analyzed on a $6 \mathrm{ft}$. $\times$ $1 / 8$ in. glass column containing $10 \%$ SP-1200 - $12 \%$ $\mathrm{H}_{3} \mathrm{PO}_{4}$ on $80 / 100$ mesh Chromosorb W-AW. After a pre-hold at $40{ }^{\circ} \mathrm{C}$ for $3 \mathrm{~min}$, the oven was programmed at $4 \%$ min to $140^{\circ} \mathrm{C}$. The injector and flame ionization detector were maintained at $160^{\circ} \mathrm{C}$, and helium flow rate was $35 \mathrm{ml} / \mathrm{min}$.

The volatile acids and phenols were analyzed on a Varian 2100 gas chromatograph and peak areas were measured with a Hewlett-Packard 3380A integrator. Using GC quantitation data obtained from analytical standards, areas for all identified components were corrected for differences in detector response.

After treatment with diazomethane, the non-volatile acids $\left(\mathrm{C}_{12}-\mathrm{C}_{36}\right)$ were analyzed with a Hewlett-Packard 5750 gas chromatograph equipped with $10 \mathrm{ft} . \times 1 / 8 \mathrm{in}$. stainless steel column containing $10 \%$ Apolar $10-\mathrm{C}$ on 100/120 Chromosorb W-AW. Temperature was programmed at $2 \% \mathrm{~min}$ from 100 to $230{ }^{\circ} \mathrm{C}$, and helium flow rate was $40 \mathrm{ml} / \mathrm{min}$. Injector and flame ionization detector were maintained at 200 and $250{ }^{\circ} \mathrm{C}$, respectively. Detector response data from a series of fatty acid methyl esters $\left(\mathrm{C}_{12}-\mathrm{C}_{82}\right)$ were plotted vs. relative retention times (relative to $C_{11}$ methyl ester). Response factors used to calculate weight yields from methyl esters were obtained from this plot. Peak areas were measured by an Autolab System IV integrator.

\section{Identification}

The phenols and acids (volatile and non-volatile) were identified by GC retention data only. The PAH in GF-C from the tobacco, PE extract, and marc pyrolyzed at 
$700^{\circ} \mathrm{C}$, and from the PE extract pyrolyzed at $850^{\circ} \mathrm{C}$ were subjected to preparative GC collection on a thermal conductivity Hewlett-Packard 5750 gas chromatograph, under conditions identical to those used to obtain analytical data. UV data were obtained on each collected GC peak. The PAH for the pyrolyzates (Table 3) were identified by UV and GC retention data $(12,13)$. When GC retention data were not known, the UV identifications were verified by mass spectral data. The PAH present in the other pyrolyzates were identified by GC retention data only. CSC PAH were identified according to Snook et al. (12).

\section{RESULTS AND DISCUSSION}

Since the majority of the PAH pyrolytic precursor studies on tobacco and its components have been performed at high temperatures, the PE extract was initially pyrolyzed at $850^{\circ} \mathrm{C}$. The pyrolyzate NF was subjected to the $\mathrm{PAH}$ analysis scheme used to analyze CSC PAH $(13,14)$. The gas chromatograms of $P A H$ present in GF-C (containing parent PAH, most of their methyl derivatives, and some multi-methyl compounds of the higher members) from CSC of Kentudky 1R1 Reference cigarettes (Figure 3) and the $850^{\circ} \mathrm{C}$ pyrolyzate of the $\mathrm{PE}$ extract (Figure 4) differed greatly in the PAH distribution (Table 3). Although higher levels of $\mathrm{PAH}$ material in the pyrolyzate NF allowed for more quantitative recovery of naphthalene and other small ring PAH (13), a significant reduction of methylated compounds was apparent in pyrolyzate vs. CSC. As expected, pyrolysis at $850^{\circ} \mathrm{C}$ favored the production of the more thermally stable parent $\mathrm{PAH}$ as well as larger ring systems. It can, therefore, be concluded, that even though the burning cone in a cigarette reaches and exceeds $850{ }^{\circ} \mathrm{C}(9)$, most of the $\mathrm{PAH}$ in cigarette smoke are produced at lower temperatures.

Table 4. Selocted PAH levels in tobacco PE extract pyrolyzates as a function of temperature.

\begin{tabular}{l|rrrrr}
\hline & \multicolumn{5}{|c}{ Amount $(\mathrm{mg} / 100 \mathrm{~g}){ }^{\mathrm{a}}$} \\
\cline { 2 - 7 } PAH & \multicolumn{5}{|c}{ Pyrolysis temperature $\left.{ }^{\circ} \mathrm{C}\right)$} \\
& 650 & 700 & 750 & 800 & 850 \\
\hline Naphthalene & 348 & 630 & 800 & 1460 & 2500 \\
Methylnaphthalenes & 709 & 1230 & 864 & 917 & 478 \\
Fluorene & 188 & 258 & 292 & 492 & 328 \\
Phenanthrene- & 212 & 326 & 664 & 1530 & 1780 \\
anthracene & & & & & \\
Methylphenanthrenes- & 389 & 582 & 736 & 760 & 378 \\
methylanthracenes & 56 & 70 & 116 & 302 & 328 \\
Fluoranthene & 102 & 132 & 232 & 534 & 686 \\
Pyrene & 119 & 155 & 220 & 240 & 136 \\
Methylpyrenes & 82 & 108 & 208 & 194 & 226 \\
$\begin{array}{l}\text { 1,2-Benzanthracene- } \\
\text { chrysene-triphenylene }\end{array}$ & & & & 288 & 336 \\
$\begin{array}{l}\text { Benzo(e)pyrene- } \\
\text { benzo(a)pyrene }\end{array}$ & 32 & 38 & 114 & 304 & 373 \\
Methylbenzopyrenes & 32 & 44 & 74 & 126 & 124 \\
\hline
\end{tabular}

a: Corrected for differences in detector response; methyl PAH assumed to respond identical to parent; average of two determinations.
Figure 5. Effects of pyrolytic temperatures on the levele of the phenanthrene fraction (PH Fr.), phenanthrene-anthrecene (PH-A), and methylphenanthrenes-anthracenes (Me PH-A) in PE extract pyrolyzates.

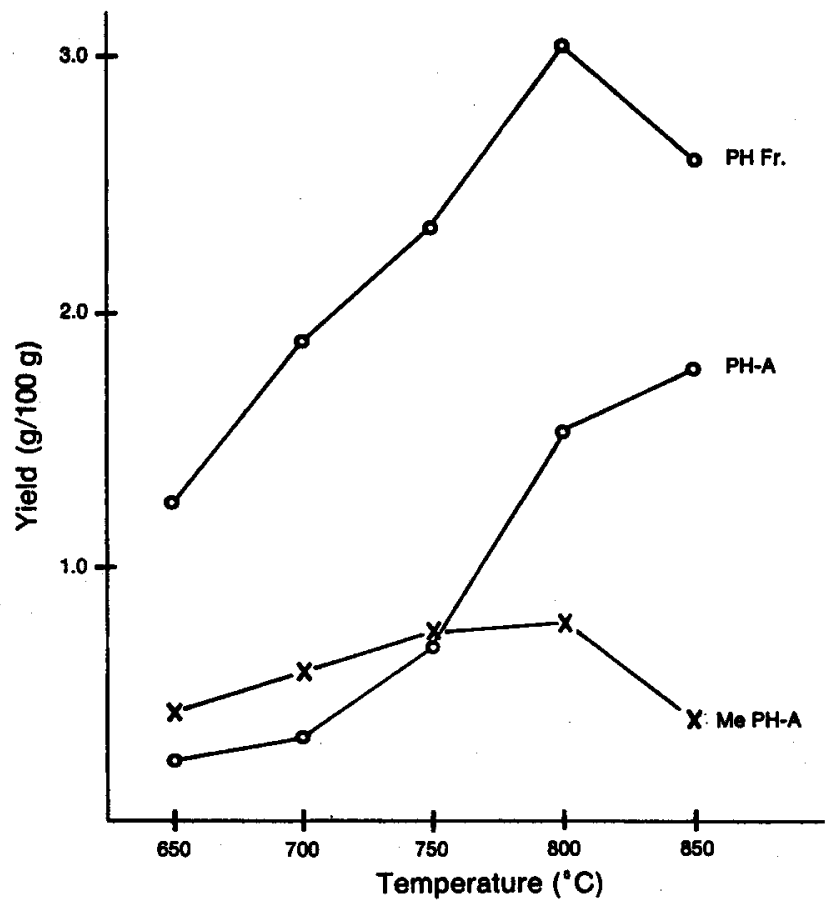

Since the PAH distribution in the $850^{\circ} \mathrm{C}$ pyrolyzate did not favorably compare with that in CSC, the $\mathrm{PE}$ extract was also pyrolyzed at $650,700,750$, and $800^{\circ} \mathrm{C}$. The yields of GC PAH fractions and of individual, selected PAH are listed in Tables 2 and 4, respectively, as functions of pyrolytic temperatures. The following general trends in product distributions were observed. The level of parent PAH increased with temperature. The level of methyl components increased until $800^{\circ} \mathrm{C}$, then declined with higher pyrolytic temperatures. The relative amounts of the high ring compounds increased with increased pyrolytic temperatures. For example, at $650^{\circ} \mathrm{C}$, the phenanthrene-anthracene to benzopyrenes ratio was about seven to one and dropped to less than five to one at $850^{\circ} \mathrm{C}$.

Examples of results can be more clearly illustrated graphically. The effect of pyrolytic temperature on phenanthrene-anthracene and the corresponding phenanthrene fraction levels is given in Figure 5. The yield of the GC phenanthrene fraction increased almost linearly with pyrolytic temperatures up to $800^{\circ} \mathrm{C}$, then decreased rapidly. The yield of parent compounds increased over

Table 5. Selected methyl PAH to parent PAH ratios in CSC and tobacco pyrolyzates.

\begin{tabular}{l|c|c|c|c}
\hline & \multicolumn{4}{|c}{ Methyl PAH to parent PAH ratios } \\
\cline { 2 - 5 } PAH & $\begin{array}{c}\text { CSC } \\
(1 \mathrm{R} 1)\end{array}$ & \multicolumn{3}{|c}{ Pyrolysis temperature $\left({ }^{\circ} \mathrm{C}\right)$} \\
\cline { 2 - 5 } & 650 & 700 & 750 \\
\hline $\begin{array}{l}\text { Phenanthrene- } \\
\text { anthracene }\end{array}$ & 2.79 & 1.52 & 1.83 & 0.94 \\
$\begin{array}{l}\text { Pyrene } \\
\begin{array}{l}\text { Benzo(e)pyrene- } \\
\text { benzo(a)pyrene }\end{array}\end{array}$ & 1.30 & 1.36 & 1.30 & 0.92 \\
\hline
\end{tabular}


Figure 3. Cas chromatogram of PAH in gel flitration cut GF-C from condeneate of 1R1 Reference clgarettes.

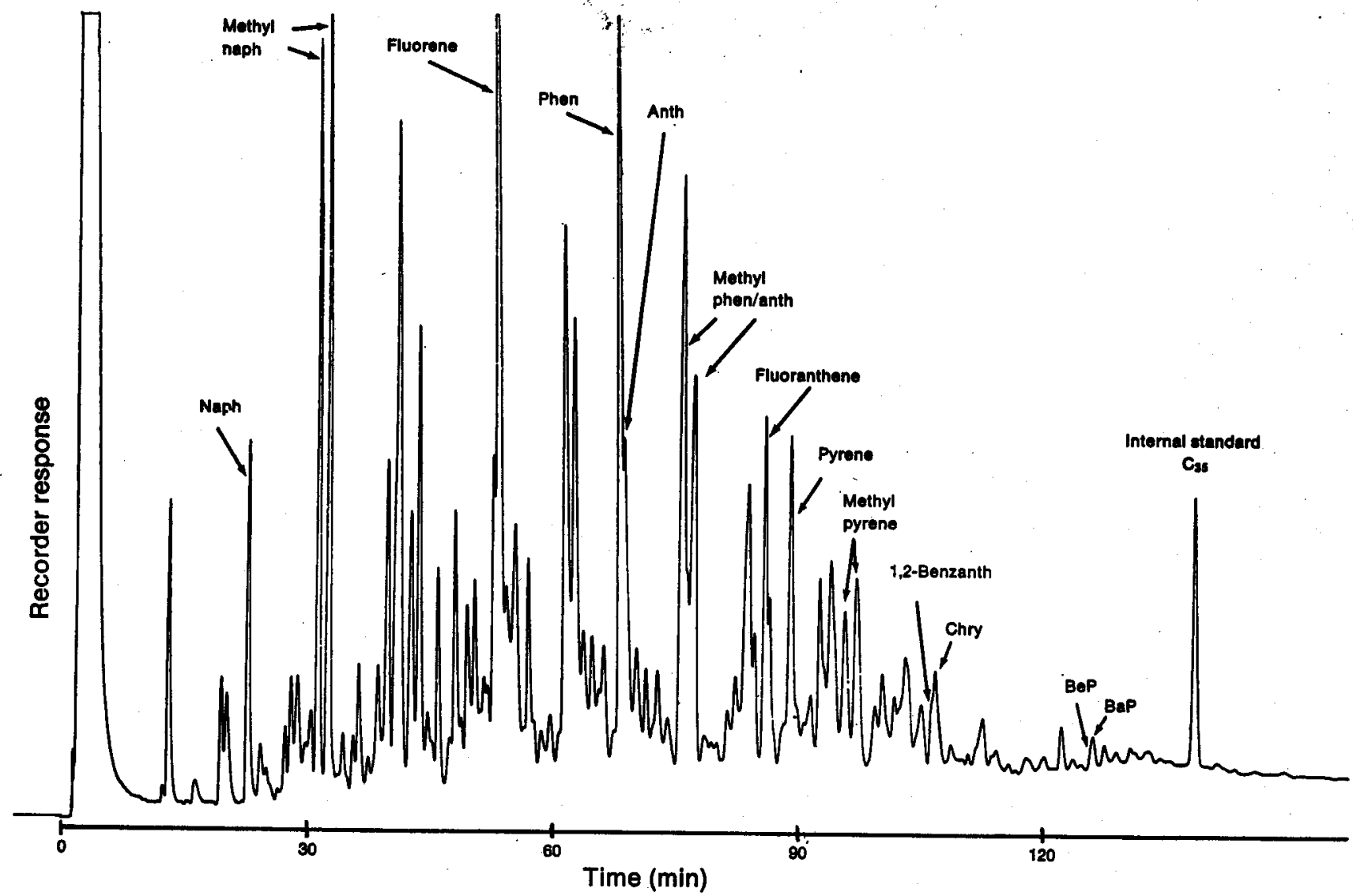

Figure 4. Gas chromatogram of PAH In gel filtration cut GF-Cin $850^{\circ} \mathrm{C}$ pyrolyzate of PE extract of fiue-cured tobacco (divided into "PAH fractions").

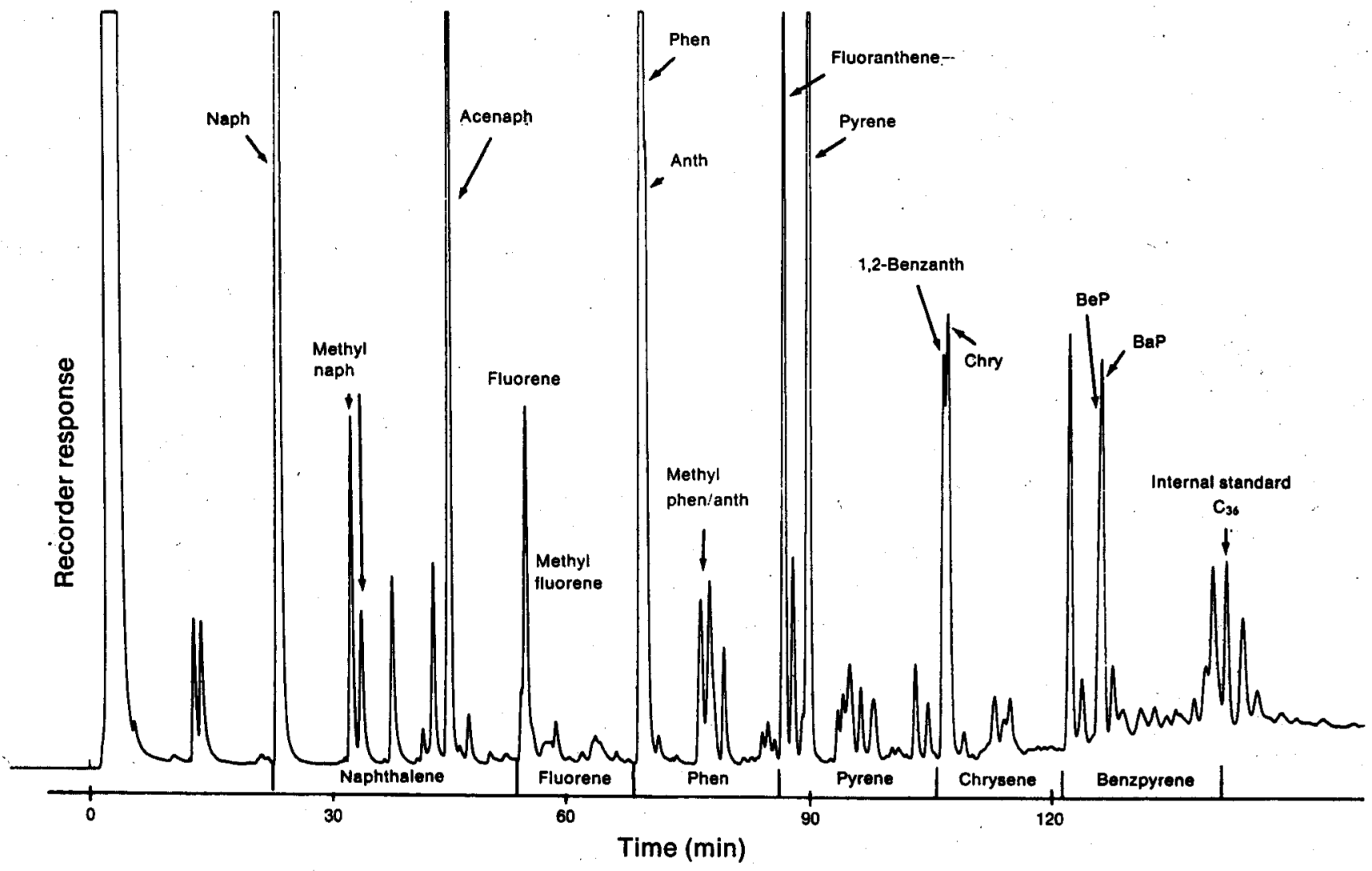


Figure 6. Effects of pyrolytic temperatures on the levels of the pyrene fraction (P Fr.), pyrene (P), and methylpyrenes (Me P) in PE extract pyrolyzates.

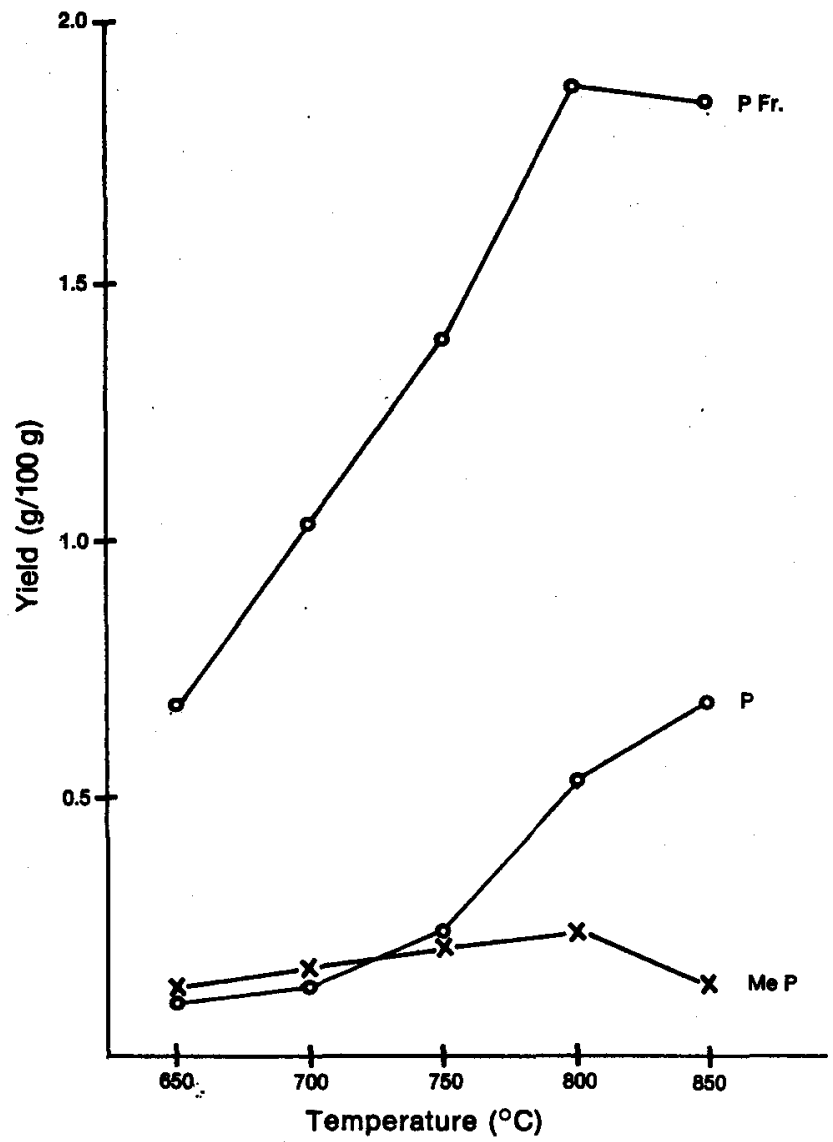

the whole range, with largest increases occurring between 700 and $800{ }^{\circ} \mathrm{C}$. The level of methyl components increased very slowly to $800{ }^{\circ} \mathrm{C}$ and then dropped off rapidly. Note the relative methyl PAH to parent $\mathrm{PAH}$ levels (Figure 5) and compare them with those of CSC (Table 5). For condensate, the methyl phenanthreneanthracene levels were considerably larger than those of their parent compounds. Therefore, the data indicated that in order to obtain a pyrolysis PAH profile similar to that for CSC, pyrolysis temperatures lower than $750^{\circ} \mathrm{C}$ should be employed. Corresponding data for the pyrene fraction are illustrated in Figure 6. Like the phenanthrene fraction, the yield of the pyrene GC fraction began to decrease at temperatures higher than $800^{\circ} \mathrm{C}$. In CSC, the methylpyrene levels were higher than those of the parent (Table 5). These data also indicated that to obtain a pyrolysis PAH profile similar to that in CSC, pyrolytic temperature should be below $750^{\circ} \mathrm{C}$. In contrast to the three- and four-ring PAH, the level of the benzopyrene fraction increased markedly with pyrolytic temperature (Figure 7). The level of methylbenzopyrenes relative to that of benzopyrenes decreased with increasing pyrolytic temperature. Because the levels of methylbenzopyrenes in CSC were about $75 \%$ of the parent PAH (Table 5), the benzopyrene data also suggested an optimum pyrolytic temperature of about $700^{\circ} \mathrm{C}$ for the PE extract.

The temperature study clearly showed that pyrolysis of the PE extract at a temperature in the range of 650 to
Figure 7. Effects of pyrolytic temperatures on the levele of the benzopyrene-fractlon (BP Fr.), benzopyrenes (BP), and methylbenzopyrenes (Me BP) in pyrolyzates of PE extract.

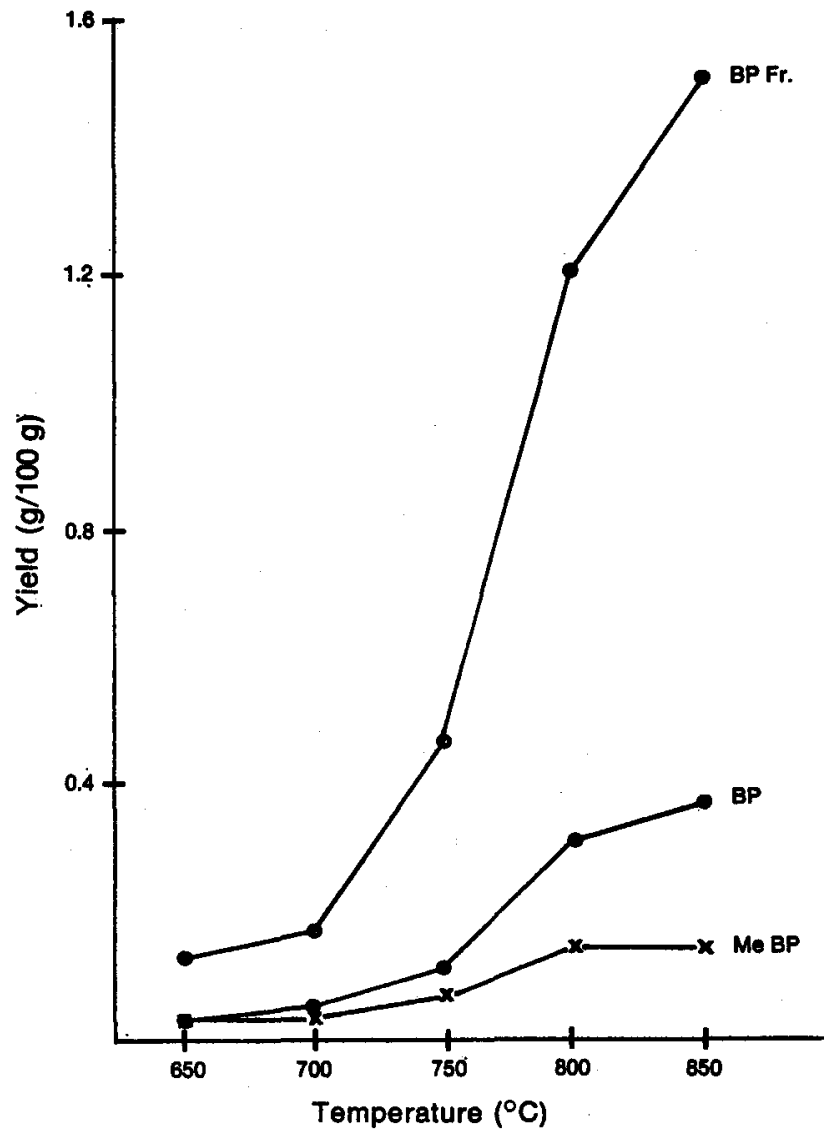

$7500^{\circ} \mathrm{C}$ in our system would yield a PAH profile most similar to that of condensate. However, the PAH components of CSC result from total reactions of all the constituents in burning tobacco. Therefore, whole tobacco was pyrolyzed at 650,700 and $750^{\circ} \mathrm{C}$, and the resulting $\mathrm{PAH}$ compared with CSC PAH of 1R1 cigarettes. The methyl PAH to parent PAH ratios for the tobacco pyrolyzates were compared to the ratios for CSC (Table 5). Again, condensate results agreed best with the 650 and $700{ }^{\circ} \mathrm{C}$ data. The relative amounts of

Table 6. Comparison of relative abundances of selected PAH In CsC and tobacco pyrolyzates.

\begin{tabular}{|c|c|c|c|c|}
\hline \multirow{3}{*}{ PAH } & \multicolumn{4}{|c|}{ Relative amounta } \\
\hline & \multirow{2}{*}{$\begin{array}{l}\text { CSC } \\
\text { (1R1) }\end{array}$} & \multicolumn{3}{|c|}{ Pyrolyzates } \\
\hline & & $650^{\circ} \mathrm{C}$ & $700^{\circ} \mathrm{C}$ & $750^{\circ} \mathrm{C}$ \\
\hline Phenanthrene-anthracene & 3.3 & 2.4 & 4.2 & 3.5 \\
\hline $\begin{array}{l}\text { Methylphenanthrenes- } \\
\text { methylanthracenes }\end{array}$ & 9.2 & 3.6 & 7.7 & 3.2 \\
\hline Fluoranthene & 0.8 & 0.5 & 0.5 & 0.6 \\
\hline Pyrene & 1.0 & 1.0 & 1.0 & 1.0 \\
\hline Methylpyrenes & 1.3 & 1.4 & 1.3 & 0.9 \\
\hline $\begin{array}{l}\text { 1,2-Benzanthracene- } \\
\text { chrysene-triphenylene }\end{array}$ & 0.5 & 0.9 & 0.8 & 0.5 \\
\hline $\begin{array}{l}\text { Benzo(e)pyrene- } \\
\text { benzo(a)pyrene }\end{array}$ & 0.2 & 0.1 & 0.3 & 0.3 \\
\hline
\end{tabular}

a: Relative to pyrene; corrected for difference in delector response. 
Figure 8. Gas chromatogram of PAH constlituents in gel filtration cut GF-C from $700^{\circ} \mathrm{C}$ tobacco pyrolyzate.

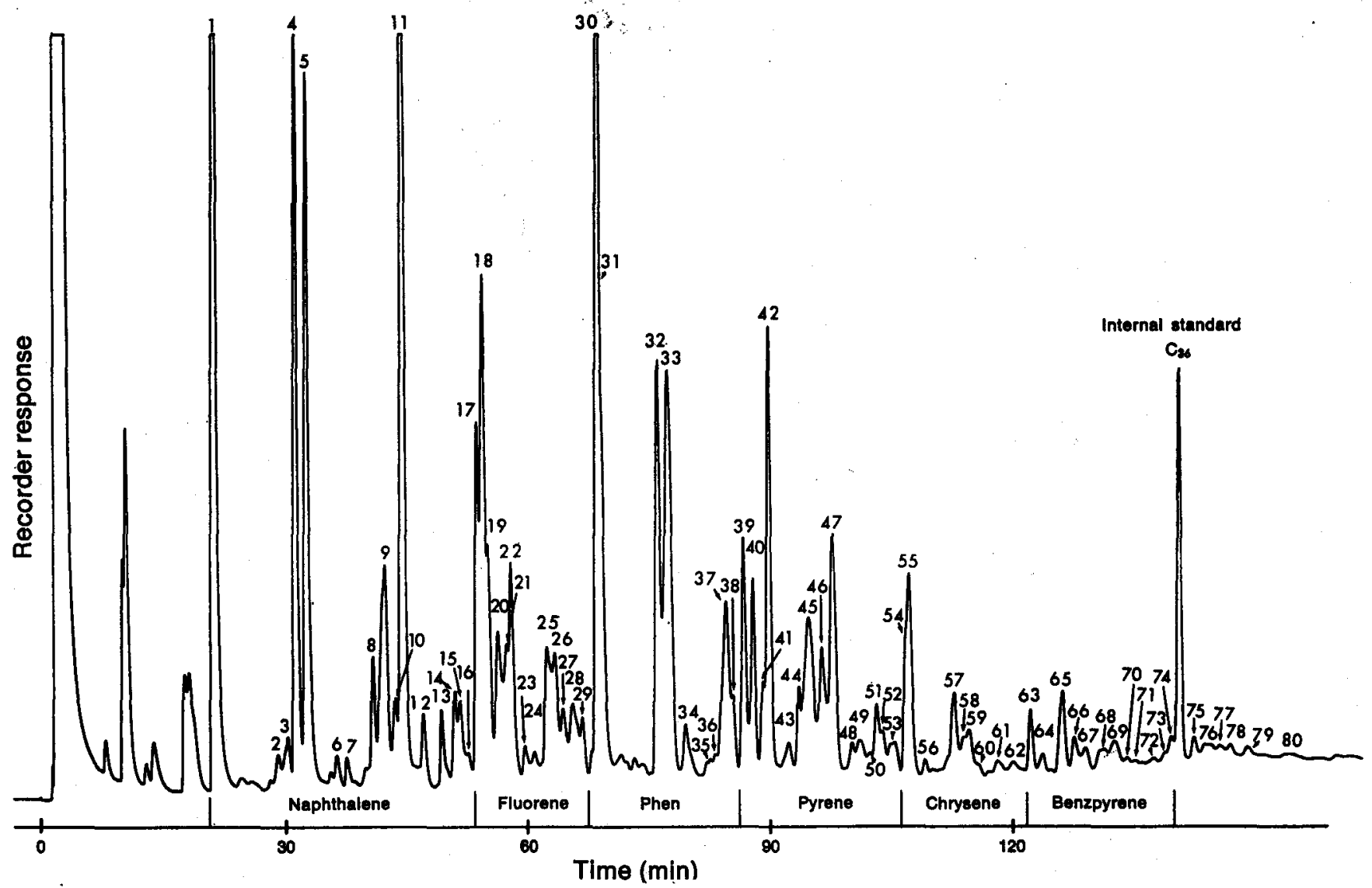

selected PAH in condensate and in tobacco pyrolyzates are compared in Table 6. At all three temperatures, the fluoranthene level was lower in all the pyrolyzates than in CSC. In agreement with the data on the pyrolyzate of the PE extract, the methyl PAH levels in the $750^{\circ} \mathrm{C}$ pyrolyzate of whole tobacco were also lower than those in CSC. The best overall compromise appeared to be pyrolysis at $700^{\circ} \mathrm{C}$. Subsequently, peak by peak comparison of the corresponding gas chromatograms showed that the GC profile of the $700{ }^{\circ} \mathrm{C}$ tobacco pyrolyzate (Figure 8) was the most similar to that of smoke condensate (Figure 3 ).

Unlike the chromatogram of the $850^{\circ} \mathrm{C} \mathrm{PE}$ extract pyrolyzate (Figure 4), that of GF-C from the tobacco pyrolyzed at $700^{\circ} \mathrm{C}$ (Figure 8) showed qualitatively all of the alkyl PAH. In the $700^{\circ} \mathrm{C}$ tobacco pyrolyzate, the dimethylnaphthalene levels (Peaks 8, 9, and 10) were lower in CSC and acenaphthylene (Peak 11) was a major component. Further comparisons showed that the $700{ }^{\circ} \mathrm{C}$ tobacco pyrolyzates contained lower levels of fluorene (Peak 18) and of fluoranthene (Peak 39). In the $700{ }^{\circ} \mathrm{C}$ pyrolyzate, there was a decrease in the amount of benzo(b,j, and $k$ )fluoranthenes (Peak 63) relative to the benzopyrenes (Peak 65), probably due to the lower fluoranthene levels in the pyrolyzate. However, it was apparent that the PAH distributions in GF-C from both condensate and pyrolyzate were similar. The gas chromatograms of the small ring, mono-, and multi-methyl PAH contained in GF-B from the CSC and $700{ }^{\circ} \mathrm{C}$ tobacco pyrolyzate are illustrated in Figures 9 and 10, respect- ively. Again, a peak by peak comparison indicates the presence of the same alkyl PAH components.

Thus, in our system, a temperature of $700{ }^{\circ} \mathrm{C}$ produced a pyrolytic PAH profile for both the whole tobacco and its PE extractables similar to that of CSC. However, the $\mathrm{PAH}$ from the PE extract were somewhat higher in alkyl $\mathrm{PAH}$ than that from tobacco. This indicated that the PE extractable material from tobacco produces not only parent PAH but also their alkyl derivatives. In order to substantiate this conclusion, we pyrolyzed the PEextracted tobacco, or marc, at $700^{\circ} \mathrm{C}$. The levels of GC PAH fractions in the $700{ }^{\circ} \mathrm{C}$ pyrolyzates from tobacco, PE extract, and marc are compared in Table 7.

Table 7. Comparison of PAH fraction levels in $700{ }^{\circ} \mathrm{C}$ pyrolyzates from tobacco, PE extract, and marc.

\begin{tabular}{l|cccc}
\hline & \multicolumn{3}{|c}{ Amounta } \\
\cline { 2 - 4 } PAH group & $\begin{array}{c}\text { Tobacco } \\
(\mathrm{mg} / 100 \mathrm{~g})\end{array}$ & $\begin{array}{c}\text { PE extractb } \\
(\mathrm{mg} / \mathbf{8} \mathrm{g})\end{array}$ & $\begin{array}{c}\text { Marcc } \\
(\mathrm{mg} / 92 \mathrm{~g})\end{array}$ \\
\hline Naphthalene & 320 & 390 & 130 \\
Fluorene & 110 & 110 & 86 \\
Phenanthrene & 160 & 150 & 78 \\
Pyrene & 63 & 82 & 35 \\
Chrysene & 18 & 26 & 8 \\
Benzopyrene & 19 & 14 & 5 \\
\hline Total & 690 & 772 & 342 \\
\hline
\end{tabular}

a: Based on GC volatile material, assuming detector response for the group identical to that of major parent PAH.

b: PE extract represents about $B \%$ of tobacco weight.

c: Marc represents about $92 \%$ of tobacco weight. 
Figure 9. Gas chromatogram of PAH constltuente in gel filtration cut GF-B of smoke condensate of 1R1 clgarettes.

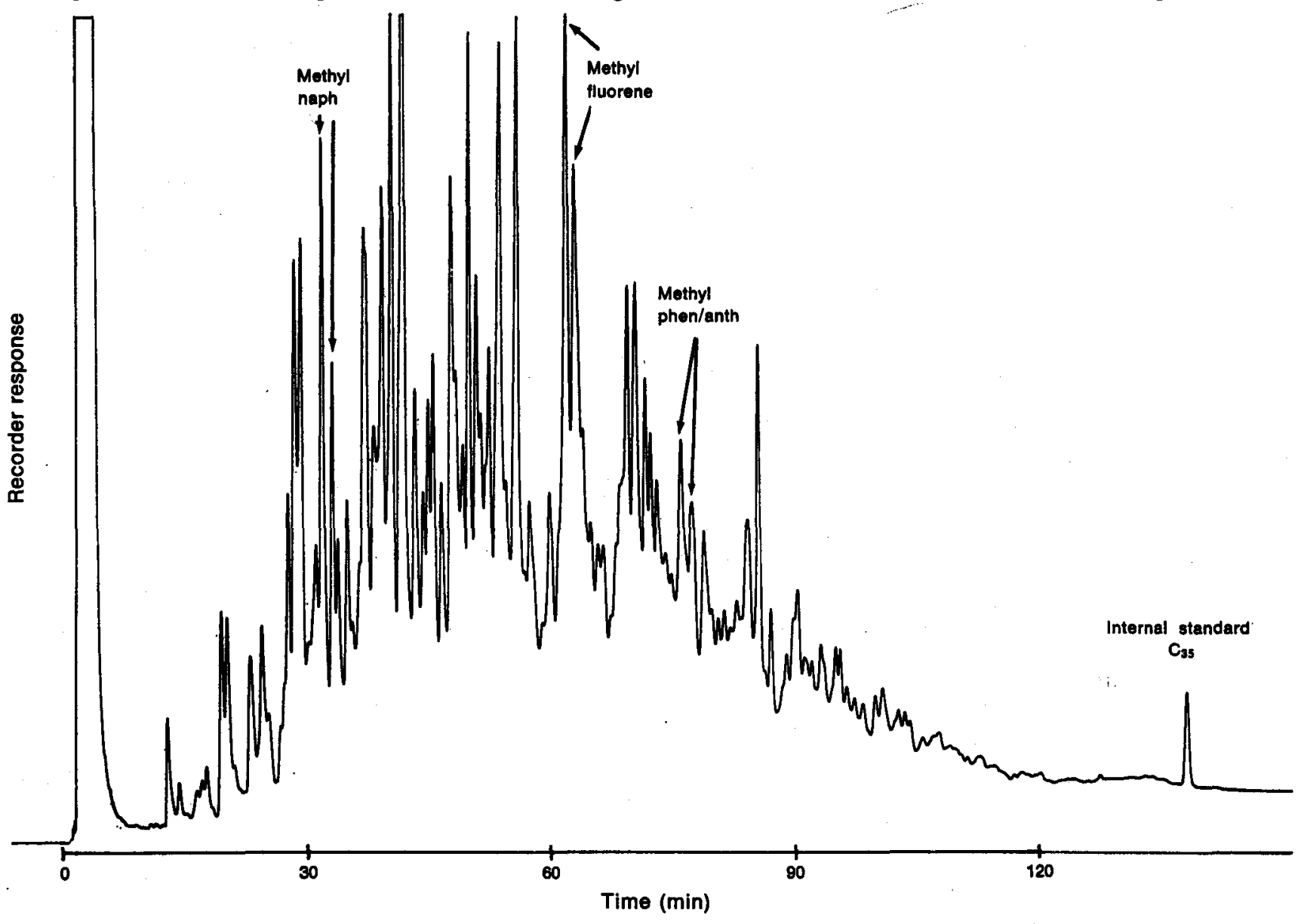

Figure 10. Gas chromatogram of PAH constituents in gel filtration cul GF-B of $700{ }^{\circ} \mathrm{C}$ tobacco pyrolyzate.

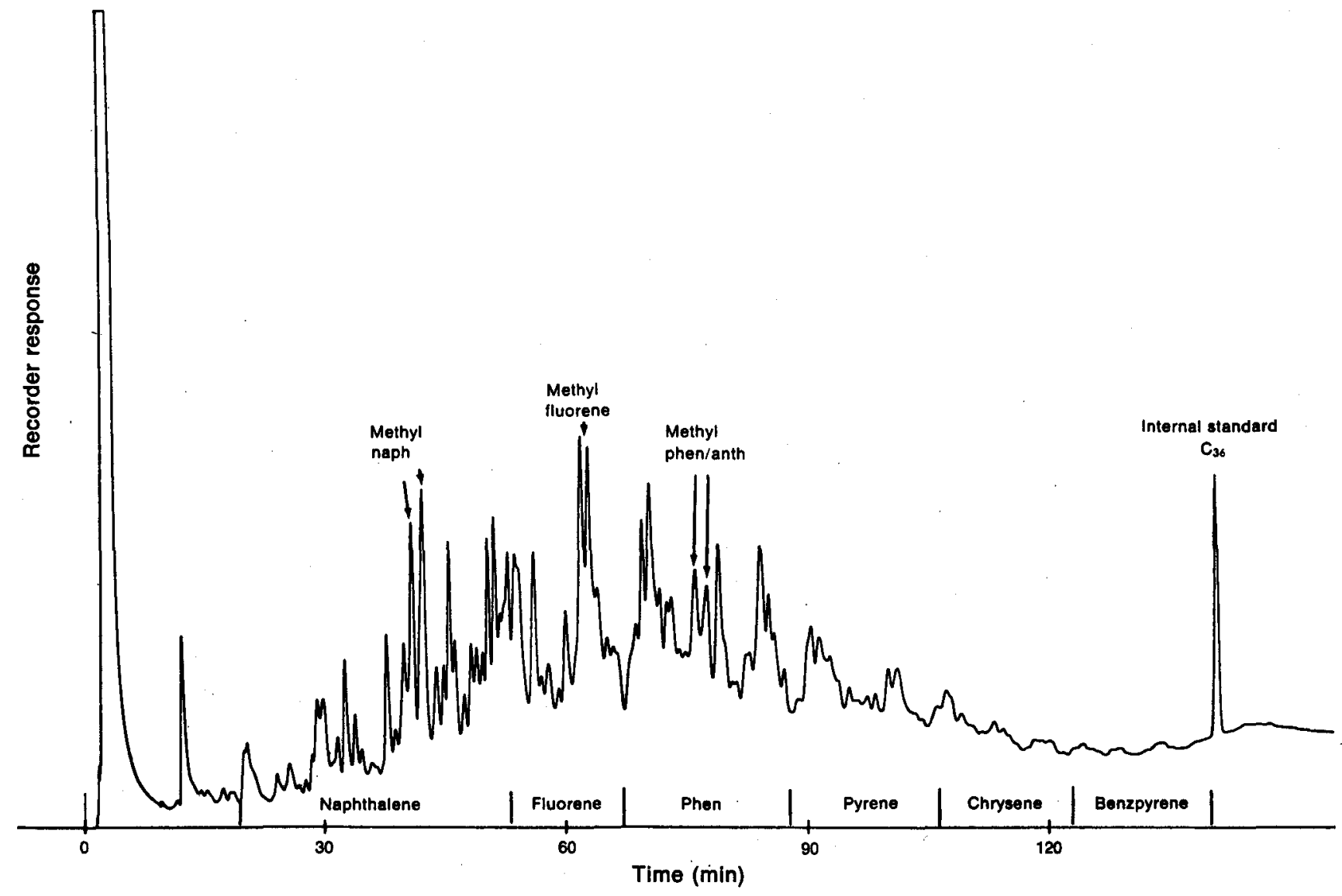


Table 8. Comparison of the phenol ylelds in $700{ }^{\circ} \mathrm{C}$ pyrolyzates of tobacco, PE extract, and marc.

:

\begin{tabular}{l|c|c|c}
\hline \multirow{2}{*}{ Phenol } & \multicolumn{3}{|c}{ Yielda } \\
\cline { 2 - 4 } & $\begin{array}{c}\text { Tobacco } \\
(\mathrm{mg} / 100 \mathrm{~g})\end{array}$ & $\begin{array}{c}\text { PE extractb } \\
(\mathrm{mg} / 8 \mathrm{~g})\end{array}$ & $\begin{array}{c}\text { Marcc } \\
(\mathrm{mg} / 92 \mathrm{~g})\end{array}$ \\
\hline Phenol & 361 & 5 & 262 \\
o-Cresol & 75 & 5 & 63 \\
m/p-Cresol & 162 & 5 & 118 \\
Ethyl- and & 91 & 13 & 70 \\
dimethyl-phenols & 16 & 3 & 11 \\
1-Naphthol & 14 & 2 & 11 \\
2-Naphthol & & 5
\end{tabular}

a: Corrected for differences in GC detector response based on one determination.

b: PE extract represents about $8 \%$ of tobacco weight.

c: Marc represents about $92 \%$ of tobacco weight.

The data are based on the relative amount of each fraction present in whole tobacco. Thus, $8 \mathrm{~g}$ of PE extract, $92 \mathrm{~g}$ of marc, and $100 \mathrm{~g}$ of tobacco are mutually equivalent. In agreement with previous work (4), PE extract produced the most PAH. The $8 \mathrm{~g}$ of extract yielded two to three times more PAH than the $92 \mathrm{~g}$ of the marc. The sum of the weights of PAH produced separately by the PE extract and the marc was considerably higher than the amount produced by the whole tobacco. This is readily explainable by the mechanisms of a series of bimolecular reactions that occur in the pyrolytic synthesis of the PAH, as proposed by Badger et al. $(1,2,3)$. Concentrating the major PAH precursors into the PE extract greatly increased the concentration of the PAH-producing intermediates in the gas phase. Since a higher concentration of reactive species greatly increases the rate of a bimolecular reaction, the pyrolysis of the PE extract would be expected to produce substantially higher levels of PAH, as observed. When tobacco is pyrolyzed, the PAH-producing intermediates, resulting from the decomposition of $\mathrm{PE}$ solubles, are diluted with other non-PAH producing products so that the probability of a PAH-forming bimolecular collision decreases. As a result, the amount of PAH produced during tobacco pyrolysis decreases.

The previous discussion on the PAH distribution in the pyrolyzates is based on the comparison of GC retention data with those PAH known to be present in CSC (12, 13). To verify that the pyrolyzates contained the same PAH components, we first fractionated, by preparative GC, the PAH in GF-C of the $700^{\circ} \mathrm{C}$ tobacco, marc, and $\mathrm{PE}$ extract and the $800^{\circ} \mathrm{C} \mathrm{PE}$ extract pyrolyzates. To facilitate the identification of individual components, we collected as many cuts as practical for each GC peak. The cuts were subjected to UV analysis, and the resulting spectra compared with those obtained for CSC PAH. The resulting component identifications are given in Table 3.

The data in Table 3 substantiated all of the previous discussions. At $700{ }^{\circ} \mathrm{C}$, a tobacco pyrolyzate PAH composition was very similar to that of CSC. The low level of methylated PAH in the marc pyrolyzate clearly shows that the PE extract is not only the major PAH
Table 9. Comparison of the acld ylelds in $700{ }^{\circ} \mathrm{C}$ pyrolyzates of tobacco, PE extract, and marc.

\begin{tabular}{|c|c|c|c|}
\hline \multirow{2}{*}{ Acid } & \multicolumn{3}{|c|}{ Ylelda } \\
\hline & $\begin{array}{c}\text { Tobacco } \\
(\mathrm{mg} / 100 \mathrm{~g})\end{array}$ & $\begin{array}{c}\text { PE extractb } \\
(\mathrm{mg} / 8 \mathrm{~g})\end{array}$ & $\begin{array}{c}\text { Marcc } \\
(\mathrm{mg} / 92 \mathrm{~g})\end{array}$ \\
\hline $\begin{array}{l}\text { Volatile acids } \\
C_{1}-C_{7} d\end{array}$ & 33 & 14 & 23 \\
\hline $\begin{array}{l}\text { Non-volatile acids } \\
\text { C }_{11-C_{34^{\circ}}}\end{array}$ & 1.2 & 1.1 & 0.4 \\
\hline
\end{tabular}

precursor, but also produces most of the methylated PAH. At $850^{\circ} \mathrm{C}$, the methyl components were not as readily formed and/or decomposed to produce high levels of the more thermally-stable parent PAH. The $700{ }^{\circ} \mathrm{C}$ marc data indicates that PE-insoluble materials are the primary precursors of oxygenated PAH, i.e. dibenzofuran (Peak 13) and naphthofuran (Peak 14) found in both the tobacco pyrolyzate and CSC.

Since the biological effects of CSC have been focused on the relationship of PAH with phenols and acid constituents $(18,19)$, it was also of interest to determine the patterns of formation of phenols and acids at the various pyrolytic temperatures. Table 8 lists the GC volatile phenols formed by the $700^{\circ} \mathrm{C}$ pyrolysis of tobacco, PE extract, and marc. In good agreement with previous work (5), the components of the marc were the major precursors of the volatile phenols. The sum of the phenolic yields from the PE extract and marc roughly equalled the yield from tobacco. Therefore, phenols appear to be formed by thermal decomposition mechanisms other than those proposed for the PAH.

Corresponding yield data for acids are presented in Table 9. Marc constituents were the major precursors of the volatile acids. Most of the high molecular weight fatty acid materials originated in the PE extract. By GC retention data, the major identifiable non-volatile acid in the tobacco and PE extract pyrolyzates was palmitic acid. The components in the marc pysplyzate could not be readily identified by $\mathrm{GC}$ retention data.

The temperature studies showed that the phenol fraction yields reached a maximum at $700^{\circ} \mathrm{C}$ and then decreased rapidly with increasing temperature. $\mathrm{GC}$ analyses revealed that phenol, cresol, ethyl phenols, and naphthol levels also reactied maximum values at $700^{\circ} \mathrm{C}$, then decreased rapidly. The acid levels remained relatively constant from 650 to $750^{\circ} \mathrm{C}$, and then dramatically decreased. According to GC retention data, the major non-volatile acids in the 650 to $750^{\circ} \mathrm{C}$ pyrolyzates were the palmitic and stearic acids. Only small amounts of the naturally occurring unsaturated acids were distilling unchanged. Above $750^{\circ} \mathrm{C}$, identification by retention data was not possible. This indicated that above $750^{\circ} \mathrm{C}$ essentially all of the naturally occurring acids in the PE extract were undergoing some degree of thermal decomposition.

In summary, we found that pyrolysis of tobacco and 
its $\mathrm{PE}$ extract at $700^{\circ} \mathrm{C}$ produced $\mathrm{PAH}$ profiles comparable to those found in CSC. Our data confirmed that the components in the PE extract were the major precursors of the PAH in smoke and that smoke phenols are primarily formed from materials not extractable with PE.

\section{SUMMARY}

Tobacco, its petroleum ether (PE) extract, and the residual extracted tobacco (marc) were pyrolyzed at $650-750^{\circ} \mathrm{C}, 650-850^{\circ} \mathrm{C}$, and $700{ }^{\circ} \mathrm{C}$, respectively. Analyses of the polynuclear aromatic hydrocarbons (PAH) produced showed that the pyrolysis of the tobacco and the $\mathrm{PE}$ extract at $700{ }^{\circ} \mathrm{C}$ produced $\mathrm{PAH}$ profiles comparable to those found in cigarette smoke. condensate (CSC). The data indicated that most of the alkyl PAH and the major PAH in cigarette smoke are derived from the PE extractables of tobacco. The constituents of the marc were the major precursors for phenols, oxygenated PAH, and low molecular weight acids; and those of $\mathrm{PE}$ extract were the major producers of high molecular weight acids.

\section{ZUSAMMENFASSUNG}

Tabak, dessen Petrolätherextrakt (PE) sowie der zurückbleibende extrahierte Tabak wurden bei Temperaturen von $650-750^{\circ} \mathrm{C}, 650-850^{\circ} \mathrm{C} \mathrm{bzw}, 700^{\circ} \mathrm{C}$ pyrolysiert. Die Analysen der entstandenen polycyclischen aromatischen Kohlenwasserstoffe (PAH) zeigten, daß die Pyrolyse des Tabaks und des Petrolätherextraktes bei $700^{\circ} \mathrm{C}$ für die polycyclischen aromatischen Kohlenwasserstoffe Profile ergibt, die den im Cigarettenrauchkondensat (CSC) gefundenen vergleichbar sind. Die Ergebnisse deuten darauf hin, daß die meisten der polycyclischen aromatischen Kohlenwasserstoffe mit Alkylgruppe und die hauptsächlichen polycyclischen aromatischen Kohlenwasserstoffe des Cigarettenrauches auf den mit Petroläther extrahierbaren Teil des Tabaks zurüdkzuführen sind. Die Bestandteile des Extraktionsrüdkstandes waren die hauptsächlichen Vorläufer der Phenole, der oxidierten polycyclischen aromatischen Kohlenwasserstoffe und der Säuren mit niedrigem Molekulargewidht; diejenigen des Petrolätherextraktes waren vorwiegend die Ursache der Säuren mit hohem Molekulargewidht.

\section{RESUME}

On a pyrolysé du tabac, l'extrait de tabac à l'éther de pétrole (PE), et le résidu de tabac extrait à $650-750^{\circ} \mathrm{C}$, $650-850^{\circ} \mathrm{C}$ et $700{ }^{\circ} \mathrm{C}$ respectivement. L'analyse des hydrocarbures polynucléaires aromatigues (PAH) produits a montré que la pyrolyse du tabac et de l'extrait $\mathrm{PE}$ à $700^{\circ} \mathrm{C}$ donne des courbes $\mathrm{PAH}$ similaires à celles du condensat de fumée (CSC). Les données recueillies indiquent que la plupart des PAH alkylés et les princi- paux PAH dans la fumée de cigarette sont dérivés des produits extractibles au PE du tabac. Les composants du. résidu de tabac extrait sont les précurseurs principaux des phénols, des PAH oxygénés et des acides à bas poids moléculaire. Les constituants de l'extrait PE sont les précurseurs principaux des acides à haut poids moléculaire.

\section{REFERENCES}

1. Badger, G. M., J. K. Donnely, and T. McL. Spotswood: Aust. J. Chem. 15 (1962) 605.

2. Badger, G. M., R. W. L. Kimber, and J. Novotny: Aust. J. Chem. 15 (1962) 616.

3. Badger, G. M., J. K. Donnely, and T. McL. Spotswood: Aust. J. Chem. 18 (1965) 1249.

4. Schlotzhaver, W. S., and I. Schmeltz: Beitr. Tabakforsch. 4 (1968) 176.

5. Schlotzhauer, W. S., O. T. Chortyk, H. C. Higman, and I. Sdhmeltz: Tob. Sci. 11 (1967) 31.

6. Wynder, E. L., G. F. Wright, and J. Lam: Cancer 11 (1958) 1140.

7. Stedman, R. L., A. P. Swain, and W. Rusaniwskyj: Tobacco 154 (1962) 20.

8. Cook, C. E., M. E. Twine, C. R. Tallent, I. Harper, G. Heunisch, J. B. Lewis, and M. E. Wall: Phytodhem. 8 (1969) 1025.

9. Wynder, E. L., and D. Hoffmann: Tobacco and tobacco smoke - Studies in experimental carcinogenesis; Academic Press, New York, 1967.

10. Tso, T. C.: Preventive Medicine 3 (1974) 294.

11. Jenkins, R. W., Jr., R. H. Newman, R. D. Carpenter, and T. S. Osdene: Beitr. Tabakforsch. 6 (1970) 295.

12. Snook, M. E., R. F. Severson, H. C. Higman, R. F. Arrendale, and O. T. Chortyk: Beitr. Tabakforsch. 8 (1976) 250.

13. Severson, R. F., M. E. Snook, O. T. Chortyk, and R. F. Arrendale: Beitr. Tabakforsch. 8 (1976) 273.

14. Severson, R. F., M. E. Snook, R. F. Arrendale, and O. T. Chortyk: Anal. Chem. 48 (1976) 1866.

15. Chortyk, O. T., R. F. Severson, and H. C. Higman: Beitr. Tabakforsch. 8 (1975) 204.

16. Pillsbury, H. C., C. C. Bright, K. J. O'Connor, and I. W. Irish: J. Assoc. Offic. Agr. Chem. 52 (1969) 458.

17. Rothwell, K. (ed.): Standard methods for the analysis of tobacco smoke; Research Paper II, Tobacco Research Council, London, 1972.

18. Bodk, F. G., A. P. Swain, and R. L. Stedman: J. Natl. Cancer Inst. 44 (1970) 1305.

19. Hoffmann, D., and E. L. Wynder: J. Natl. Cancer Inst. 52 (1971) 613.

The authors' address:

United States Department of Agriculture,

Agricultural Research Service,

Tobacco and Health Laboratory,

P.O. Box 5677 ,

Athens, Georgia, 30604, U.S.A. 